\title{
Balanced Metric and Berezin Quantization on the Siegel-Jacobi Ball
}

\author{
Stefan BERCEANU
}

National Institute for Physics and Nuclear Engineering, Department of Theoretical Physics, PO BOX MG-6, Bucharest-Magurele, Romania

E-mail: Berceanu@theory.nipne.ro

URL: http://www.theory.nipne.ro/index.php/mcp-home

Received March 03, 2016, in final form June 17, 2016; Published online June 27, 2016

http://dx.doi.org/10.3842/SIGMA.2016.064

\begin{abstract}
We determine the matrix of the balanced metric of the Siegel-Jacobi ball and its inverse. We calculate the scalar curvature, the Ricci form and the Laplace-Beltrami operator of this manifold. We discuss several geometric aspects related with Berezin quantization on the Siegel-Jacobi ball.
\end{abstract}

Key words: Jacobi group; Siegel-Jacobi ball; balanced metric; homogenous Kähler manifolds; Laplace-Beltrami operator; scalar curvature; Ricci form; Berezin quantization

2010 Mathematics Subject Classification: 32Q15; 81S10; 53D50; 57Q35; 81R30

\section{Introduction}

The Jacobi groups are semidirect products of appropriate semisimple real algebraic groups of Hermitian type with Heisenberg groups [23, 33, 49, 74]. As unimodular, nonreductive, algebraic groups of Harish-Chandra type [68], the Jacobi groups are intensively studied in mathematics $[15,22,72,73,74,76,78,79]$. The Siegel-Jacobi (partially bounded [78, 79]) domains are homogeneous manifolds associated to the Jacobi groups by the generalized Harish-Chandra embedding. It was underlined $[10,11,12]$ that the Siegel-Jacobi disk is a nonsymmetric, reductive [63], quantizable [66], Q-K. Lu Kähler manifold [54], not Einstein with respect to the balanced metric $[1,31]$, with constant negative scalar curvature. In the present paper we investigate similar geometric properties for the Siegel-Jacobi ball.

As was already emphasized [5, 7, 9], the Jacobi group is relevant in several branches of physics, as: quantum mechanics, geometric quantization, quantum optics, nuclear structure, signal processing [14, 48, 65, 70]. In particular, the Jacobi group is responsible [8] for the squeezed states in quantum optics [58]. Recently, new significant applications of the Jacobi group have been underlined in various domains as: quantum tomography [59], quantum teleportation [30], Vlasov kinetic equation [35], general symmetry methods for analyzing solutions of differential equations using Lie's prolongation method [40, 69], establishing the link between the standard Gaussian distribution and the Siegel-Jacobi space [60]. Berezin's quantization and Berezin symbols related to the Jacobi group have been also investigated [25, 26].

The real Jacobi group of index $n$ is defined as $G_{n}^{J}(\mathbb{R})=\mathrm{H}_{n}(\mathbb{R}) \rtimes \mathrm{Sp}(n, \mathbb{R})$, where $\mathrm{H}_{n}(\mathbb{R})$ is the real Heisenberg group of real dimension $(2 n+1)[9,76]$. Let $g=(M, X, k), g^{\prime}=\left(M^{\prime}, X^{\prime}, k^{\prime}\right) \in$ $G_{n}^{J}(\mathbb{R})$, where $X=(\lambda, \mu) \in \mathbb{R}^{2 n}$ and $(X, k) \in \mathrm{H}_{n}(\mathbb{R})$. Then the composition law in $G_{n}^{J}(\mathbb{R})$ is

$$
g g^{\prime}=\left(M M^{\prime}, X M^{\prime}+X^{\prime}, k+k^{\prime}+X M^{\prime} J X^{\prime t}\right),
$$

where

$$
J=\left(\begin{array}{cc}
\mathbb{O}_{n} & \mathbb{1}_{n} \\
-\mathbb{1}_{n} & \mathbb{O}_{n}
\end{array}\right)
$$


Also it is considered the restricted real Jacobi group $G_{n}^{J}(\mathbb{R})_{0}$, consisting only of elements of the form above, but $g=(M, X)$.

To the Jacobi group $G_{n}^{J}(\mathbb{R})$ it is associated the homogeneous manifold - the Siegel-Jacobi upper half-plane $-X_{n}^{J} \approx \mathbb{C}^{n} \times X_{n}$, where the Siegel upper half-plane $X_{n}=\operatorname{Sp}(n, \mathbb{R}) / \mathrm{U}(n)$ is realized as

$$
X_{n}:=\left\{V \in M(n, \mathbb{C}) \mid V=S+\mathrm{i} R, S, R \in M(n, \mathbb{R}), R>0, S^{t}=S, R^{t}=R\right\} .
$$

The (complex version of the) Jacobi group of index $n$ is defined as $G_{n}^{J}=\mathrm{H}_{n} \rtimes \operatorname{Sp}(n, \mathbb{R})_{\mathbb{C}}$, where $\mathrm{H}_{n}$ denotes the $(2 n+1)$-dimensional Heisenberg group [7, 9, 76]. The composition law is

$$
\left(g_{1}, \alpha_{1}, t_{1}\right)\left(g_{2}, \alpha_{2}, t_{2}\right)=\left(g_{1} g_{2}, g_{2}^{-1} \times \alpha_{1}+\alpha_{2}, t_{1}+t_{2}+\Im\left(g_{2}^{-1} \times \alpha_{1} \bar{\alpha}_{2}\right)\right),
$$

where $\alpha_{i} \in \mathbb{C}^{n}, t_{i} \in \mathbb{R}$ and $g_{i} \in \operatorname{Sp}(n, \mathbb{R})_{\mathbb{C}}, i=1,2$ have the form

$$
g=\left(\begin{array}{ll}
p & q \\
\bar{q} & \bar{p}
\end{array}\right), \quad p, q \in M(n, \mathbb{C}),
$$

and $g \times \alpha=p \alpha+q \bar{\alpha}$, and $g^{-1} \times \alpha=p^{*} \alpha-q^{t} \bar{\alpha}$.

The Siegel-Jacobi ball, denoted $\mathcal{D}_{n}^{J}[7]$, is the homogeneous manifold associated with the Jacobi group $G_{n}^{J}$, whose points are in $\mathbb{C}^{n} \times \mathcal{D}_{n}$, where $\mathcal{D}_{n}$ denotes the Siegel (open) ball. The non-compact hermitian symmetric $\operatorname{space} \operatorname{Sp}(n, \mathbb{R})_{\mathbb{C}} / \mathrm{U}(n)$ admits a matrix realization as a bounded homogeneous domain:

$$
\mathcal{D}_{n}:=\left\{W \in M(n, \mathbb{C}): W=W^{t}, N>0, N:=\mathbb{1}_{n}-W \bar{W}\right\} .
$$

$\mathcal{D}_{n}$ is a hermitian symmetric space of type CI (cf. [36, Table V, p. 518]), identified with the symmetric bounded domain of type II, $\mathfrak{R}_{\mathrm{II}}$ in Hua's notation [38].

In $[6,7,9]$ we have attached coherent states (CS) [64] to the Jacobi group $G_{n}^{J}$ with support on the Siegel-Jacobi ball $\mathcal{D}_{n}^{J}$. The particular case of coherent states attached to the Jacobi group $G_{1}^{J}$ defined on the Siegel-Jacobi disk $\mathcal{D}_{1}^{J}$ has been investigated in $[5,10]$. In the present paper we don't use effectively the coherent states, but we use results obtained in geometry using the coherent state approach. The homogeneous Kähler two-form on $\mathcal{D}_{n}^{J}$, denoted $\omega_{\mathcal{D}_{n}^{J}}$, has been obtained in $[7,9]$ from the scalar product of two coherent state vectors, and this will be the starting point of the present investigation. The reproducing kernel function for the SiegelJacobi ball was obtained previously, see [62, p. 532] and references there. We recall that in [5] we have shown that when expressed in variables on $x_{1}$ obtained (via the partial Cayley transform) from variables on $\mathcal{D}_{1}^{J}$, the Kähler two-form $\omega_{X_{1}^{J}}$ is the one determined by Berndt [21, 23] and Kähler [42, 43]. Applying the partial Cayley transform, which we recall later in Proposition 2.5, from $\omega_{X_{n}^{J}}$ obtained in [77], it was obtained the homogeneous Kähler two-form on the SiegelJacobi ball $\omega_{\mathcal{D}_{n}^{J}}$ - in fact, Yang considers a Jacobi group more general than $G_{n}^{J}$. In [9] we have shown that when expressed in appropriate variables, $\omega_{\mathcal{D}_{n}^{J}}$ is a particular case of that obtained by Yang in [79]. In [12] we have underlined that the homogeneous metric corresponding to $\omega_{\mathcal{D}_{1}^{J}}$ is a balanced metric $[1,31]$. In the present paper it is emphasized that the metric corresponding to $\omega_{\mathcal{D}_{n}^{J}}[6,7,9]$ is the balanced metric. We also point out several geometric aspects related with Berezin quantization on the Siegel-Jacobi ball.

The paper is laid out as follows. In Section 2 we recall several notions which are used in the paper. The notion of balanced metric on homogeneous Kähler manifolds is briefly recalled in Section 2.1. Also other notions which appear in connection with Berezin quantization, as quantizable manifold, Berezin kernel, CS-type group, Q.-K. Lu manifold and diastasis are briefly recalled. The homogenous metric on the Siegel upper half plane and Siegel ball, which we need later, are recalled in Section 2.2. We have considered useful to collect in Section 2.3 several 
formulas referring to the differential geometry of the Siegel-Jacobi disk $\mathcal{D}_{1}^{J}$, a guide for the formulas deduced in the present paper for the Siegel-Jacobi ball $\mathcal{D}_{n}^{J}$. Section 3 contains the original results of the present paper. The balanced metric is obtained from the Kähler potential, calculated previously [7] using the coherent states. Do to the symmetry of the variables $W \in M(n, \mathbb{C})$ describing points on the Siegel ball $\mathcal{D}_{n}$, we write down the matrix associated with the metric $h_{\mathcal{D}_{n}^{J}}(z, W)$ on the Siegel-Jacobi ball as a four-blocks matrix expressed in $(z, W) \in \mathbb{C}^{n} \times \mathcal{D}_{n}$, but also in a variable $\eta$ related with $(z, W)$ by $\eta=\left(\mathbb{1}_{n}-W \bar{W}\right)^{-1}(z+W \bar{z})$. The significance of the change of coordinates $\mathrm{FC}:(\eta, W) \rightarrow(z, W)$ as a homogeneous Kähler diffeomorphism was underlined in [9] in the context of the fundamental conjecture [32] for homogeneous Kähler manifolds (Gindikin and Vinberg [75]). In order to determine the inverse of the metric matrix attached to the metric $h_{\mathcal{D}_{n}^{J}}$, we need the "inverse" of the metric matrix $\mathfrak{h}_{\mathcal{D}_{n}}^{k}$ of the Siegel ball, presented in Section 2.2 - the complication comparatively with that on the noncompact Grassmann manifold [3] comes from the fact the matrices describing the points on Siegel ball are symmetric - see Lemma 2.7. $\left(h_{\mathcal{D}_{n}}^{k}\right)^{-1}$ is calculated in Proposition B.2, where more details on the calculation of the Laplace-Beltrami operator on Siegel ball are given. Theorem 3.2 contains the matrix of the metric of the Siegel-Jacobi ball and its "inverse" (in the sense of Lemma 2.7). Proposition 3.3 collects a description of the geometry of the Siegel-Jacobi ball from the point of view of Berezin's quantization. In Section 3.2 it is shown that the scalar curvature of the Siegel-Jacobi ball is constant and negative. This is compatible with Theorem 4.1 in [51] which asserts that a Kähler manifold that admits a regular quantization has constant scalar curvature. It is also shown that $\mathcal{D}_{n}^{J}$ is not an Einstein manifold with respect to the balanced metric, but it is one with respect to the Bergman metric. Section 3.3 contains the Laplace-Beltrami operator on $\mathcal{D}_{n}^{J}$. Remark A.1 essentially asserts that if the metric on the homogeneous space in $M=G / H$ is invariant to the action of $G$ on $M$, then the same is true for the Laplace-Beltrami operator, a fact used in the proof of Theorem 3.2. In Appendix A we reproduce also a lemma which calculates the invariant volume of the Siegel-Jacobi ball used in [7]. In Appendix B already mentioned are presented auxiliary calculations to the paper [57] of Maass and a remark concerning the results obtained in [39] referring to the Laplace-Beltrami operator on the Siegel ball and Siegel upper half plane.

The main new results of the present paper are contained in Remark 2.3, Lemma 2.7, Theorem 3.2, Propositions 3.3, 3.4 and 3.5. They generalize to the Siegel-Jacobi ball the results recalled in Section 2.3 obtained for the Siegel-Jacobi disk.

Notation. We denote by $\mathbb{R}, \mathbb{C}, \mathbb{Z}$, and $\mathbb{N}$ the field of real numbers, the field of complex numbers, the ring of integers, and the set of non-negative integers, respectively. We denote the imaginary unit $\sqrt{-1}$ by $\mathrm{i}$, and the real and imaginary part of a complex number by $\Re$ and respectively $\Im$, i.e., we have for $z \in \mathbb{C}, z=\Re z+\mathrm{i} \Im z$, and $\bar{z}=c c(z)=\Re z-\mathrm{i} \Im z . M(m \times n, \mathbb{F}) \approx \mathbb{F}^{m n}$ denotes the set of all $m \times n$ matrices with entries in the field $\mathbb{F} . \quad M(n \times 1, \mathbb{F})$ is identified with $\mathbb{F}^{n}$. Set $M(n, \mathbb{F}):=M(n \times n, \mathbb{F})$. For any $A \in M_{n}(\mathbb{F}), A^{t}$ denotes the transpose matrix of $A$. For $A \in M_{n}(\mathbb{C}), \bar{A}$ denotes the conjugate matrix of $A$ and $A^{*}=\bar{A}^{t}$. For $A \in M_{n}(\mathbb{C})$, the inequality $A>0$ means that $A$ is positive definite. The identity matrix of degree $n$ is denoted by $\mathbb{1}_{n}$ and $\mathbb{O}_{n}$ denotes the $M(n, \mathbb{F})$-matrix with all entries 0 . We consider a complex separable Hilbert space $\mathfrak{H}$ endowed with a scalar product which is antilinear in the first argument, $(\lambda x, y)=\bar{\lambda}(x, y), x, y \in \mathfrak{H}, \lambda \in \mathbb{C} \backslash 0$. If $A$ is a linear operator, we denote by $A^{\dagger}$ its adjoint. If $\pi$ is representation of a Lie group $G$ on the Hilbert space $\mathfrak{H}$ and $\mathfrak{g}$ is the Lie algebra of $G$, we denote $\boldsymbol{X}:=\mathrm{d} \pi(X)$ for $X \in \mathfrak{g}$. A complex analytic manifold is Kählerian if it is endowed with a Hermitian metric whose imaginary part $\omega$ has $\mathrm{d} \omega=0$ [36]. We denote the action of a Lie group $G$ on the space $M$ by $G \times M \rightarrow M$. A coset space $M=G / H$ is homogenous Kählerian if it caries a Kählerian structure invariant under the group $G$ [24]. By a Kähler homogeneous diffeomorphism we mean a diffeomorphism $\phi: M \rightarrow N$ of homogeneous Kähler manifolds such that $\phi^{*} \omega_{N}=\omega_{M}$. We use Einstein convention that repeated indices are implicitly summed over. 
If the Kähler-two form has the local expression

$$
\omega_{M}(z)=\mathrm{i} \sum_{\alpha, \beta=1}^{n} h_{\alpha \bar{\beta}}(z) \mathrm{d} z_{\alpha} \wedge \mathrm{d} \bar{z}_{\beta}, \quad h_{\alpha \bar{\beta}}=\bar{h}_{\beta \bar{\alpha}}=h_{\bar{\beta} \alpha}
$$

we denote

$$
h^{\alpha \bar{\beta}}:=\left(h_{\alpha \bar{\beta}}\right)^{-1},
$$

i.e., we have

$$
h_{\alpha \bar{\epsilon}} h^{\epsilon \bar{\beta}}=\delta_{\alpha \beta} .
$$

In this paper we use the following expression for the Laplace-Beltrami operator on Kähler manifolds $M$ with the Kähler two-form (1.3):

$$
\Delta_{M}(z):=\sum_{\alpha, \beta=1}^{n}\left(h_{\alpha \bar{\beta}}\right)^{-1} \frac{\partial^{2}}{\partial \bar{z}_{\alpha} \partial z_{\beta}}
$$

cf., e.g., Lemma 3 in the Appendix of [18] or in [41, equation (5.2.15), p. 253], where the Laplace-Beltrami operator is $-\Delta_{M}(z)$ and the author uses the convention $h^{i j} h_{k \bar{j}}=\delta_{i j}$ (see [41, equation (5.2.14), p. 252]) instead of our notation (1.4), (1.5). This means that $h^{l \bar{k}}$ in Jost's notation corresponds to $h^{k \bar{l}}=\left(h_{k \bar{l}}\right)^{-1}$ in our notation.

\section{Preliminaries}

\subsection{Balanced metric and Berezin quantization via coherent states}

We consider a $G$-invariant Kähler two-form $\omega_{M}(1.3)$ on the $2 n$-dimensional homogeneous manifold $M=G / H$ derived from the Kähler potential $f(z, \bar{z})[29]$

$$
h_{\alpha \bar{\beta}}=\frac{\partial^{2} f}{\partial z_{\alpha} \partial \bar{z}_{\beta}} .
$$

As was underlined in [12] for $\mathcal{D}_{1}^{J}$, the homogeneous hermitian metric determined in $[5,7,9]$ is in fact a balanced metric, because it corresponds to the Kähler potential calculated as the product of two CS-vectors

$$
f(z, \bar{z})=\ln K_{M}(z, \bar{z}), \quad K_{M}(z, \bar{z})=\left(e_{\bar{z}}, e_{\bar{z}}\right) .
$$

In the approach of Perelomov [64] to CS, it is supposed that there exists a continuous, unitary, irreducible representation $\pi$ of a Lie group $G$ on the separable complex Hilbert space $\mathfrak{H}$. The coherent vector mapping $\varphi$ is defined locally, on a coordinate neighborhood $\mathcal{V}_{0} \subset M$ (cf. [4, 13]):

$$
\varphi: M \rightarrow \overline{\mathfrak{H}}, \quad \varphi(z)=e_{\bar{z}},
$$

where $\overline{\mathfrak{H}}$ denotes the Hilbert space conjugate to $\mathfrak{H}$. The vectors $e_{\bar{z}} \in \overline{\mathfrak{H}}$ indexed by the points $z \in M$ are called Perelomov's CS vectors. Using Perelomov's CS vectors, we consider Berezin's approach to quantization on Kähler manifolds with the supercomplete set of vectors verifying the Parceval overcompletness identity $[17,18,19,20]$

$$
\left(\psi_{1}, \psi_{2}\right)_{\mathcal{F}_{K}}=\int_{M}\left(\psi_{1}, e_{\bar{z}}\right)\left(e_{\bar{z}}, \psi_{2}\right) \mathrm{d} \nu_{M}(z, \bar{z}), \quad \psi_{1}, \psi_{2} \in \mathfrak{H},
$$




$$
\mathrm{d} \nu_{M}(z, \bar{z})=\frac{\Omega_{M}(z, \bar{z})}{\left(e_{\bar{z}}, e_{\bar{z}}\right)}, \quad \Omega_{M}:=\frac{1}{n !} \underbrace{\omega \wedge \cdots \wedge \omega}_{n \text { times }} .
$$

The reproducing kernel for the Hilbert space of holomorphic, square integrable functions with respect to the scalar product of the type (2.3) was calculated via CS as the scalar product $K_{M}(z, \bar{w})=\left(e_{\bar{z}}, e_{\bar{w}}\right)[5,7,9]$.

On the other side, let us consider the weighted Hilbert space $\mathfrak{H}_{f}$ of square integrable holomorphic functions on $M$, with weight $\mathrm{e}^{-f}[34]$

$$
\mathfrak{H}_{f}=\left\{\left.\phi \in \operatorname{hol}(M)\left|\int_{M} \mathrm{e}^{-f}\right| \phi\right|^{2} \Omega_{M}<\infty\right\} .
$$

In order to identify the Hilbert space $\mathfrak{H}_{f}$ defined by $(2.4)$ with the Hilbert $\mathcal{F}_{K}$ space with scalar product $(2.3)$, we have to consider the $\epsilon$-function $[27,66,67]$

$$
\epsilon(z)=\mathrm{e}^{-f(z)} K_{M}(z, \bar{z}) .
$$

If the Kähler metric on the complex manifold $M$ is obtained from the Kähler potential via (1.3), (2.1) and (2.2) is such that $\epsilon(z)$ is a positive constant, then the metric is called balanced. This denomination was firstly used in [31] for compact manifolds, then it was used in [1] for noncompact manifolds and also in [52] in the context of Berezin quantization on homogeneous bounded domains.

The balanced hermitian metric of $M$ in local coordinates is

$$
\mathrm{d} s_{M}^{2}(z, \bar{z})=\sum_{\alpha, \beta=1}^{n} \frac{\partial^{2}}{\partial z_{\alpha} \partial \bar{z}_{\beta}} \ln \left(K_{M}(z, \bar{z})\right) \mathrm{d} z_{\alpha} \otimes \mathrm{d} \bar{z}_{\beta}, \quad K_{M}(z, \bar{z})=\left(e_{\bar{z}}, e_{\bar{z}}\right) .
$$

We recall that in $[27,66,67]$ Berezin's quantization via coherent states was globalized and extended to non-homogeneous manifolds in the context of geometric (pre-)quantization [47]. To the Kähler manifold $(M, \omega)$, it is also attached the triple $\sigma=(\mathcal{L}, h, \nabla)$, where $\mathcal{L}$ is a holomorphic (prequantum) line bundle on $M, h$ is the hermitian metric on $\mathcal{L}$ and $\nabla$ is a connection compatible with metric and the Kähler structure [16]. The manifold is called quantizable if the curvature of the connection [29] $F(X, Y)=\nabla_{X} \nabla_{Y}-\nabla_{Y} \nabla_{X}-\nabla_{[X, Y]}$ has the property that $F=-\mathrm{i} \omega_{M}$, or $\partial \bar{\partial} \log \hat{h}=\mathrm{i} \omega_{M}$, where $\hat{h}$ is a local representative of $h$. Then $\omega_{M}$ is integral, i.e., $c_{1}[\mathcal{L}]=\left[\omega_{M}\right]$. The reproducing (weighted Bergman) kernel admits the series expansion

$$
K_{M}(z, \bar{w}) \equiv\left(e_{\bar{z}}, e_{\bar{w}}\right)=\sum_{i=0}^{\infty} \varphi_{i}(z) \bar{\varphi}_{i}(w),
$$

where $\Phi=\left(\varphi_{0}, \varphi_{1}, \ldots\right)$ is an orthonormal base with respect to the scalar product $(2.3)$ :

$$
\int_{M} \bar{\varphi}_{i} \varphi_{j} \frac{\Omega_{M}}{K_{M}}=\delta_{i j}, \quad i, j \in \mathbb{N} .
$$

The base $\Phi$ is finite-dimensional for compact manifolds $M$.

Let $\xi: \mathfrak{H} \backslash 0 \rightarrow \mathbb{P}(\mathfrak{H})$ be the canonical projection $\xi(\boldsymbol{z})=[\boldsymbol{z}]$. The Fubini-Study metric in the nonhomogeneous coordinates $[z]$ is the hermitian metric on $\mathbb{C P}^{\infty}$ (see [44] for details)

$$
\left.\mathrm{d} s^{2}\right|_{\mathrm{FS}}([z])=\frac{(\mathrm{d} \boldsymbol{z}, \mathrm{d} \boldsymbol{z})(\boldsymbol{z}, \boldsymbol{z})-(\mathrm{d} \boldsymbol{z}, \boldsymbol{z})(\boldsymbol{z}, \mathrm{d} \boldsymbol{z})}{(\boldsymbol{z}, \boldsymbol{z})^{2}} .
$$

It was proved [67] (see also [12]) that: 
Remark 2.1. If $\epsilon(z)$ is constant on $M$, then the balanced Hermitian metric on $M$ is the pullback

$$
\mathrm{d} s_{M}^{2}(z)=\iota_{M}^{*} \mathrm{~d} s_{\mathrm{FS}}^{2}(z)=\mathrm{d} s_{\mathrm{FS}}^{2}\left(\iota_{M}(z)\right)
$$

of the Fubini-Study metric (2.7) via the embedding

$$
\iota_{M}: M \hookrightarrow \mathbb{C P}^{\infty}, \quad \iota_{M}(z)=\left[\varphi_{0}(z): \varphi_{1}(z): \ldots\right] .
$$

If $M$ is a compact manifold, the embedding (2.9) is the Kodaira embedding.

If the homogeneous Kähler manifold $M=G / H$ to which we associate the Hilbert space of functions $\mathcal{F}_{K}$ with respect to the scalar product $(2.3)$ admits a holomorphic embedding $\iota_{M}: M \hookrightarrow$ $\mathbb{P}\left(\mathcal{H}^{\infty}\right)$, then $M$ is called a CS-orbit, and $G$ is called a CS-type group [4, 50, 62].

We denote the normalized Bergman kernel (the two-point function of $M[2,16]$ ) by

$$
\kappa_{M}\left(z, \bar{z}^{\prime}\right):=\frac{K_{M}\left(z, \bar{z}^{\prime}\right)}{\sqrt{K_{M}(z) K_{M}\left(z^{\prime}\right)}}=\left(\tilde{e}_{\bar{z}}, \tilde{e}_{\bar{z}^{\prime}}\right)=\frac{\left(e_{\bar{z}}, e_{\bar{z}^{\prime}}\right)}{\left\|e_{\bar{z}}\right\|\left\|e_{\bar{z}^{\prime}}\right\|} .
$$

The set $\Sigma_{z}:=\left\{z^{\prime} \in M \mid \kappa_{M}\left(z, \bar{z}^{\prime}\right)=0\right\}$ was called [2, 16] polar divisor relative to $z \in M$, while a manifold for which $\Sigma_{z}=\varnothing, \forall z \in M$ was called in [12] a Q.-K. Lu manifold, extending to manifolds a denomination introduced for domains in $\mathbb{C}^{n}$ [54]. Note that for a particular class of compact homogeneous manifolds that includes the hermitian symmetric spaces, $\Sigma_{z}$ is equal with the cut locus relative to $z \in M$ (see the definition of the cut locus, e.g., [46, p. 100]), and $\Sigma_{z}$ is a divisor in the sense of algebraic geometry $[2,16]$.

By Berezin kernel $b_{M}: M \times M \rightarrow[0,1] \in \mathbb{R}$, in this paper we mean:

$$
b_{M}\left(z, z^{\prime}\right):=\left|\kappa_{M}\left(z, \bar{z}^{\prime}\right)\right|^{2} .
$$

The Calabi's diastasis [28] expressed via the CS-vectors [27] reads

$$
D_{M}\left(z, z^{\prime}\right):=-\ln b_{M}\left(z, z^{\prime}\right)=-2 \ln \left|\left(\tilde{e}_{\bar{z}}, \tilde{e}_{\bar{z}^{\prime}}\right)\right| \text {. }
$$

Generalizing a theorem proved for homogeneous bounded domains [52], in [53] Loi and Mossa have proved:

Theorem 2.2. Let $(M, \omega)$ be a simply-connected homogeneous Kähler manifold such that the associated Kähler form $\omega$ is integral. Then there exists a constant $\mu_{0}>0$ such that $M$ equipped with $\mu_{0} \omega$ is projectively induced.

With Theorem 2.2 and the recalled definitions, we can formulate the

Remark 2.3. Let $M=G / H$ be a simply-connected homogeneous Kähler manifold. Then the following assertions are equivalent:

A) $M$ is a quantizable Kähler manifold,

B) $M$ admits a balanced metric,

C) $M$ is CS-type manifold and $G$ is a CS-type group,

D) $M$ is projectively induced and we have (2.8), (2.9).

Loi and Mossa have proved [53] the following

Theorem 2.4. Let $(M, \omega)$ be a homogeneous Kähler manifold. Then the following are equivalent:

a) $M$ is contractible, 
b) $(M, \omega)$ admits a global Kähler potential,

c) $(M, \omega)$ admits a global diastasis $D_{M}: M \times M \rightarrow \mathbb{R}$,

d) $(M, \omega)$ admits a Berezin quantization.

The proof of the Theorem 2.4 is based on the fundamental conjecture for homogeneous bounded domains [32] and the asymptotic of the Bergman kernel [34].

Theorems 2.2, 2.4 and Remark 2.3 will be used in the proof of Proposition 3.3.

\subsection{Elements of geometry of the Siegel upper half-plane and Siegel ball}

Firstly we recall some standard facts about the symplectic group and its algebra, see references and details in $[7,9]$. Even if we will be concerned mainly with the Jacobi group $G_{n}^{J}$, we recall some basic facts about Cayley and partial Cayley transform which will appear in the paper.

\subsubsection{The Cayley transform}

If $X \in \mathfrak{s p}(n, \mathbb{R})$, i.e., $X^{t} J+J X=0$, then

$$
X=\left(\begin{array}{cc}
a & b \\
c & -a^{t}
\end{array}\right), \quad \text { where } \quad b=b^{t}, \quad c=c^{t}, \quad a, b, c \in M(n, \mathbb{R}) .
$$

If $g=\left(\begin{array}{ll}a & b \\ c & d\end{array}\right) \in \operatorname{Sp}(n, \mathbb{R})$, i.e., $g^{t} J g=J$, then the matrices $a, b, c, d \in M(n, \mathbb{R})$ have the properties

$$
a^{t} c=c^{t} a, \quad b^{t} d=d^{t} b, \quad a^{t} d-c^{t} b=\mathbb{1}_{n}, \quad a b^{t}=b a^{t}, \quad c d^{t}=d c^{t}, \quad a d^{t}-b c^{t}=\mathbb{1}_{n} .
$$

We have the correspondence

$$
g=\left(\begin{array}{ll}
a & b \\
c & d
\end{array}\right) \in M(2 n, \mathbb{R}) \leftrightarrow g_{\mathbb{C}}=\mathfrak{C}^{-1} g \mathfrak{C}=\left(\begin{array}{ll}
p & q \\
\bar{q} & \bar{p}
\end{array}\right), \quad p, q \in M(n, \mathbb{C}),
$$

where

$$
\begin{aligned}
& \mathcal{C}=\left(\begin{array}{cc}
\mathrm{i} \mathbb{1}_{n} & \mathrm{i} \mathbb{1}_{n} \\
-\mathbb{1}_{n} & \mathbb{1}_{n}
\end{array}\right), \quad \mathrm{C}^{-1}=\frac{1}{2}\left(\begin{array}{cc}
-\mathrm{i} \mathbb{1}_{n} & -\mathbb{1}_{n} \\
-\mathrm{i} \mathbb{1}_{n} & \mathbb{1}_{n}
\end{array}\right), \\
& 2 a=p+q+\bar{p}+\bar{q}, \quad 2 b=\mathrm{i}(\bar{p}-\bar{q}-p+q), \quad 2 c=\mathrm{i}(p+q-\bar{p}-\bar{q}), \\
& 2 d=p-q+\bar{p}-\bar{q}, \quad 2 p=a+d+\mathrm{i}(b-c), \quad 2 q=a-d-\mathrm{i}(b+c) .
\end{aligned}
$$

To every $g \in \operatorname{Sp}(n, \mathbb{R})$, we associate via $(2.12) g \mapsto g_{\mathbb{C}} \in \operatorname{Sp}(n, \mathbb{R})_{\mathbb{C}} \equiv \operatorname{Sp}(n, \mathbb{C}) \cap \mathrm{U}(n, n)$ as in $(1.1)$, where the matrices $p, q \in M(n, \mathbb{C})$ have the properties

$$
p p^{*}-q q^{*}=\mathbb{1}_{n}, \quad p q^{t}=q p^{t}, \quad p^{*} p-q^{t} \bar{q}=\mathbb{1}_{n}, \quad p^{t} \bar{q}=q^{*} p .
$$

Let us consider an element $h=(g, l)$ in $G_{n}^{J}(\mathbb{R})_{0}$, i.e.,

$$
g=\left(\begin{array}{ll}
a & b \\
c & d
\end{array}\right) \in \operatorname{Sp}(n, \mathbb{R}), \quad l=(n, m) \in \mathbb{R}^{2 n},
$$

and $V \in \mathcal{X}_{n}, u \in \mathbb{C}^{n} \equiv \mathbb{R}^{2 n}$.

Let $g \in \operatorname{Sp}(n, \mathbb{R})_{\mathbb{C}}$ be of the form (1.1), (2.14) and $\alpha, z \in \mathbb{C}^{n}$. The (transitive) action $(g, \alpha) \times(W, z)=\left(W_{1}, z_{1}\right)$ of the Jacobi group $G_{n}^{J}$ on the Siegel-Jacobi ball $\mathcal{D}_{n}^{J}$ is given by the formulas $[7]$

$$
W_{1}=(p W+q)(\bar{q} W+\bar{p})^{-1}=\left(W q^{*}+p^{*}\right)^{-1}\left(q^{t}+W p^{t}\right),
$$




$$
z_{1}=\left(W q^{*}+p^{*}\right)^{-1}(z+\alpha-W \bar{\alpha}) .
$$

Now we consider the partial Cayley transform [7, 9] $\Phi: X_{n}^{J} \rightarrow \mathcal{D}_{n}^{J}, \Phi(V, u)=(W, z)$

$$
W=\left(V-\mathrm{i} \mathbb{1}_{n}\right)\left(V+\mathrm{i} \mathbb{1}_{n}\right)^{-1}, \quad z=2 \mathrm{i}\left(V+\mathrm{i} \mathbb{1}_{n}\right)^{-1} u,
$$

with the inverse partial Cayley transform $\Phi^{-1}: \mathcal{D}_{n}^{J} \rightarrow X_{n}^{J}, \Phi^{-1}(W, z)=(V, u)$

$$
V=\mathrm{i}\left(\mathbb{1}_{n}-W\right)^{-1}\left(\mathbb{1}_{n}+W\right), \quad u=\left(\mathbb{1}_{n}-W\right)^{-1} z .
$$

Let us define $\Theta: G_{n}^{J}(\mathbb{R})_{0} \rightarrow\left(G_{n}^{J}\right)_{0}, \Theta(h)=h_{*}, h=(g, n, m), h_{*}=\left(g_{\mathbb{C}}, \alpha\right)$.

We have proved in $[9$, Proposition 2] (see also $[15,78]$ )

Proposition 2.5. $\Theta$ is an group isomorphism and the action of $\left(G_{n}^{J}\right)_{0}$ on $\mathcal{D}_{n}^{J}$ is compatible with the action of $G_{n}^{J}(\mathbb{R})_{0}$ on $X_{n}^{J}$ through the biholomorphic partial Cayley transform (2.17), i.e., if $\Theta(h)=h_{*}$, then $\Phi h=h_{*} \Phi$. More exactly, if the action of $\left(G_{n}^{J}\right)_{0}$ on $\mathcal{D}_{n}^{J}$ is given by (2.16), then the action of $G_{n}^{J}(\mathbb{R})_{0}$ on $X_{n}^{J}$ is given by $(g, l) \times(V, u) \rightarrow\left(V_{1}, u_{1}\right) \in X_{n}^{J}$, where

$$
\begin{aligned}
& V_{1}=(a V+b)(c V+d)^{-1}=\left(V c^{t}+d^{t}\right)^{-1}\left(V a^{t}+b^{t}\right), \\
& u_{1}=\left(V c^{t}+d^{t}\right)^{-1}(u+V n+m) .
\end{aligned}
$$

The matrices $g$ in (2.15) and $g_{\mathbb{C}}$ in (1.1) are related by (2.12), (2.13), while $\alpha=m+\mathrm{i}$, $m, n \in \mathbb{R}^{n}$.

\subsubsection{The metric}

Siegel has determined the metric on $X_{n}, \operatorname{Sp}(n, \mathbb{R})$-invariant to the action (2.19) (see [71, equation (2)] or [37, Theorem 3, p. 644]):

$$
\mathrm{d} s_{\chi_{n}}^{2}(R)=\operatorname{Tr}\left(R^{-1} \mathrm{~d} V R^{-1} \mathrm{~d} \bar{V}\right) .
$$

With the Cayley transform (2.18) and the relations

$$
\dot{V}=2 \mathrm{i} U \dot{W} U, \quad U=\left(\mathbb{1}_{n}-W\right)^{-1}, \quad 2 R=\left(\mathbb{1}_{n}+W\right) U+\left(\mathbb{1}_{n}+\bar{W}\right) \bar{U},
$$

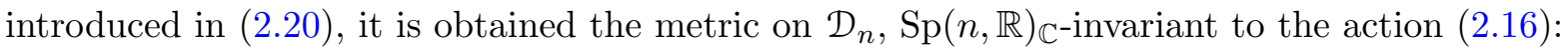

$$
\mathrm{d} s_{\mathcal{D}_{n}}^{2}(W)=4 \operatorname{Tr}(M \mathrm{~d} W \bar{M} \mathrm{~d} \bar{W}), \quad W \in \mathcal{D}_{n}, \quad M=\left(\mathbb{1}_{n}-W \bar{W}\right)^{-1},
$$

associated with the Kähler two-form (3.26), modulo the factor $\frac{2}{k}$.

Remark 2.6. The metric (2.22) can be written as in (2.24), (2.25).

Proof. Equation (2.22) can be written down as

$$
\begin{aligned}
& \frac{1}{4} \mathrm{~d} s_{\mathcal{D}_{n}}^{2}(W)=\sum_{p, q, m, n} H_{p q \bar{m} \bar{n}} \mathrm{~d} w_{p q} \mathrm{~d} \bar{w}_{m n}, \\
& H_{p q \bar{m} \bar{n}}:=M_{n p} M_{m q}, \quad M:=\left(\mathbb{1}_{n}-W \bar{W}\right)^{-1} .
\end{aligned}
$$

We have

$$
\begin{aligned}
\frac{1}{4} \mathrm{~d} s_{\mathcal{D}_{n}}^{2}(W)= & \sum_{p<q ; m<n}\left(H_{p q \bar{m} \bar{n}}+H_{p q \bar{n} \bar{m}}+H_{q p \bar{m} \bar{n}}+H_{q p \bar{n} \bar{m}}\right) \mathrm{d} w_{p q} \mathrm{~d} \bar{w}_{m n} \\
& +\sum_{p<q}\left(H_{p q \bar{m} \bar{m}}+H_{q p \bar{m} \bar{m}}\right) \mathrm{d} w_{p q} \mathrm{~d} \bar{w}_{m m}
\end{aligned}
$$




$$
+\sum_{m<n}\left(H_{p p \bar{m} \bar{n}}+H_{p p \bar{n} \bar{m}}\right) \mathrm{d} w_{p p} \mathrm{~d} \bar{w}_{m n}+\sum H_{p p \bar{m} \bar{m}} \mathrm{~d} w_{p p} \mathrm{~d} \bar{w}_{m m}
$$

For reasons which will be clarified further (see formulas (3.24), (3.18e)), we write (2.23) as

$$
\frac{1}{4} \mathrm{~d} s_{\mathcal{D}_{n}}^{2}(W)=\sum_{p \leq q ; m \leq n} h_{p q m n}^{k} \mathrm{~d} w_{p q} \mathrm{~d} \bar{w}_{m n}
$$

where

$$
h_{p q \bar{m} \bar{n}}^{k}=2 M_{m p} M_{n q}\left(1-\delta_{p q}\right)+2 M_{m q} M_{n p}\left(1-\delta_{m n}\right)+M_{m p}^{2} \delta_{p q} \delta_{m n},
$$

which proves the statement of the remark.

In order to take into account the symmetry of the matrix $W=\left(w_{i j}\right)_{i, j=1, \ldots, n}$, we introduce the notation (see [9, equation (4.5)]):

$$
\Delta_{p q}^{i j}:=\frac{\partial w_{i j}}{\partial w_{p q}}=\delta_{i p} \delta_{j q}+\delta_{i q} \delta_{j p}-\delta_{i j} \delta_{p q} \delta_{i p}, \quad w_{i j}=w_{j i} .
$$

For calculation of the "inverse" $h^{-1}$ of the metric matrix $h$ of $\mathcal{D}_{n}^{J}$ - see Theorem 3.2 - we need the "inverse" of the matrix (2.25), which we calculate in Proposition B.2, exploiting the formula given in [57] for the Laplace-Beltrami operator (1.6) on $\mathcal{X}_{n}$ with the result (see (B.11)):

$$
k_{m n \bar{u} \bar{v}}=\frac{1}{2}\left(N_{v n} \bar{N}_{m u}+N_{v m} \bar{N}_{n u}\right), \quad N=\mathbb{1}_{n}-W \bar{W} .
$$

We calculate

$$
E_{p q}^{u v}:=\sum_{m \leq n} h_{p q \bar{m} \bar{n}}^{k} k_{m n \bar{u} \bar{v}} .
$$

We shall prove a relation which gives the sense of the "inverse" matrix of (2.25), important in Theorem 3.2:

Lemma 2.7. The "inverse" matrix of the metric matrix (2.25) on the Siegel ball $\mathcal{D}_{n}$ is the matrix (2.27) and we have

$$
E_{p q}^{u v}=\Delta_{p q}^{u v}
$$

Proof. a) Firstly, we consider the case $p=q$ in (2.28). We get successively

$$
\begin{aligned}
E_{p p}^{u v}= & \sum_{m \leq n}\left(2-\delta_{m n}\right)\left(\mathbb{1}_{n}-W \bar{W}\right)_{m p}^{-1}\left(\mathbb{1}_{n}-\bar{W} W\right)_{p n}^{-1} k_{m n \bar{u} \bar{v}} \\
= & \left(\mathbb{1}_{n}-W \bar{W}\right)_{m p}^{-1}\left(\mathbb{1}_{n}-\bar{W} W\right)_{p m}^{-1}\left(\mathbb{1}_{n}-W \bar{W}\right)_{v m}\left(\mathbb{1}_{n}-\bar{W} W\right)_{m u} \\
& +\sum_{m<n}\left(\mathbb{1}_{n}-W \bar{W}\right)_{m p}^{-1}\left(\mathbb{1}_{n}-\bar{W} W\right)_{p n}^{-1} \\
& \times\left[\left(\mathbb{1}_{n}-W \bar{W}\right)_{v n}\left(\mathbb{1}_{n}-\bar{W} W\right)_{m u}+\left(\mathbb{1}_{n}-W \bar{W}\right)_{v m}\left(\mathbb{1}_{n}-\bar{W} W\right)_{n u}\right] \\
= & \left(\mathbb{1}_{n}-W \bar{W}\right)_{m p}^{-1}\left(\mathbb{1}_{n}-\bar{W} W\right)_{p m}^{-1}\left(\mathbb{1}_{n}-W \bar{W}\right)_{v m}\left(\mathbb{1}_{n}-\bar{W} W\right)_{m u} \\
& +\sum_{m<n}\left(\mathbb{1}_{n}-W \bar{W}\right)_{n p}^{-1}\left(\mathbb{1}_{n}-\bar{W} W\right)_{p m}^{-1}\left(\mathbb{1}_{n}-W \bar{W}\right)_{v n}\left(\mathbb{1}_{n}-\bar{W} W\right)_{m u} \\
& +\sum_{n<m}\left(\mathbb{1}_{n}-W \bar{W}\right)_{m p}^{-1}\left(\mathbb{1}_{n}-\bar{W} W\right)_{p m}^{-1}\left(\mathbb{1}_{n}-W \bar{W}\right)_{v n}\left(\mathbb{1}_{n}-\bar{W} W\right)_{m u} \\
= & \delta_{u p} \delta_{v p} .
\end{aligned}
$$


b) Now we consider the case $p \neq q$ in (2.28). We get successively

$$
\begin{aligned}
E_{p q}^{u v}= & 2 \sum_{m \leq n}\left[\left(\mathbb{1}_{n}-W \bar{W}\right)_{m p}^{-1}\left(\mathbb{1}_{n}-\bar{W} W\right)_{q n}^{-1}+\left(\mathbb{1}_{n}-W \bar{W}\right)_{m q}^{-1}\left(\mathbb{1}_{n}-\bar{W} W\right)_{p n}^{-1}\left(1-\delta_{m n}\right)\right] k_{m n \bar{u} \bar{v}} \\
= & 2\left(\mathbb{1}_{n}-W \bar{W}\right)_{m p}^{-1}\left(\mathbb{1}_{n}-\bar{W} W\right)_{q m}^{-1}\left(\mathbb{1}_{n}-W \bar{W}\right)_{v m}\left(\mathbb{1}_{n}-\bar{W} W\right)_{m u} \\
& +\sum_{m<n}\left[\left(\mathbb{1}_{n}-W \bar{W}\right)_{m p}^{-1}\left(\mathbb{1}_{n}-\bar{W} W\right)_{q n}^{-1}+\left(\mathbb{1}_{n}-W \bar{W}\right)_{m q}^{-1}\left(\mathbb{1}_{n}-\bar{W} W\right)_{p n}^{-1}\right] \\
& \times\left[\left(\mathbb{1}_{n}-W \bar{W}\right)_{v n}\left(\mathbb{1}_{n}-\bar{W} W\right)_{v n}\left(\mathbb{1}_{n}-\bar{W} W\right)_{m u}+\left(\mathbb{1}_{n}-W \bar{W}\right)_{v m}\left(\mathbb{1}_{n}-\bar{W} W\right)_{n u}\right] \\
= & \delta_{p u} \delta_{v q}+\delta_{p v} \delta_{q u} .
\end{aligned}
$$

Putting together (2.30) and (2.31), with formula

$$
E_{u v}^{p q}=E_{u v}^{p q}\left(1-\delta_{p q}\right)+E_{u v}^{p q} \delta_{p q}
$$

we have proved (2.29).

\subsection{Geometry of the Siegel-Jacobi disk}

We recall some formulas describing the differential geometry of the Siegel-Jacobi disk $\mathcal{D}_{1}^{J}[5$, $10,12]$.

The (transitive) action of the group $G_{1}^{J}=\mathrm{H}_{1} \rtimes \mathrm{SU}(1,1) \ni(g, \alpha) \times(z, w) \rightarrow\left(z_{1}, w_{1}\right) \in \mathcal{D}_{1}^{J}$ on the Siegel disk is given by the formulas

$$
w_{1}=\frac{a w+b}{\delta}, \quad \delta=\bar{b} w+\bar{a}, \quad \mathrm{SU}(1,1) \ni g=\left(\begin{array}{cc}
a & b \\
\bar{b} & \bar{a}
\end{array}\right)
$$

where $|a|^{2}-|b|^{2}=1$,

$$
z_{1}=\frac{\gamma}{\delta}, \quad \gamma=z+\alpha-\bar{\alpha} w
$$

The balanced Kähler two-form is obtained with formulas (1.3), (2.1)

$$
-\mathrm{i} \omega_{k \mu}(z, w)=h_{z \bar{z}} \mathrm{~d} z \wedge \mathrm{d} \bar{z}+h_{z \bar{w}} \mathrm{~d} z \wedge \mathrm{d} \bar{w}-h_{\bar{z} w} \mathrm{~d} \bar{z} \wedge \mathrm{d} w+h_{w \bar{w}} \mathrm{~d} w \wedge \mathrm{d} \bar{w}
$$

where the Kähler potential (2.2) is

$$
f(z, w)=\mu \frac{2 z \bar{z}+z^{2} \bar{w}+\bar{z}^{2} w}{2 P}-2 k \ln (P), \quad P=1-w \bar{w} .
$$

$k$ indexes the positive discrete series of $\mathrm{SU}(1,1)(2 k \in \mathbb{N})$, while $\mu>0$ indexes the representations of the Heisenberg group.

Proposition 2.8. The balanced Kähler two-form $\omega_{k \mu}$ on $\mathcal{D}_{1}^{J}, G_{1}^{J}$-invariant to the action (2.32), (2.33), can be written as

$$
\begin{aligned}
& -\mathrm{i} \omega_{k \mu}(z, w)=2 k \frac{\mathrm{d} w \wedge \mathrm{d} \bar{w}}{P^{2}}+\mu \frac{\mathcal{A} \wedge \overline{\mathcal{A}}}{P}, \quad \mathcal{A}=\mathrm{d} z+\bar{\eta}(z, w) \mathrm{d} w \\
& z=\eta-w \bar{\eta}, \quad \text { and } \quad \eta=\eta(z, w):=\frac{z+\bar{z} w}{1-w \bar{w}} .
\end{aligned}
$$

The matrix of the balanced metric $(2.5) h=h(\varsigma), \varsigma:=(z, w) \in \mathbb{C} \times \mathcal{D}_{1}$, determined with the Kähler potential (2.34), reads

$$
h(\varsigma):=\left(\begin{array}{cc}
h_{z \bar{z}} & h_{z \bar{w}} \\
h_{w \bar{z}}=\bar{h}_{z \bar{w}} & h_{w \bar{w}}
\end{array}\right)=\left(\begin{array}{cc}
\frac{\mu}{P} & \mu \frac{\eta}{P} \\
\mu \frac{\bar{\eta}}{P} & \frac{2 k}{P^{2}}+\mu \frac{|\eta|^{2}}{P}
\end{array}\right) .
$$


The inverse of the matrix (2.35) reads

$$
h^{-1}(\varsigma)=\left(\begin{array}{ll}
h^{z \bar{z}} & h^{z \bar{w}} \\
h^{w \bar{z}} & h^{w \bar{w}}
\end{array}\right)=\left(\begin{array}{cc}
\frac{P}{\mu}+\frac{P^{2}|\eta|^{2}}{2 k} & -\frac{P^{2} \eta}{2 k} \\
-\frac{P^{2} \bar{\eta}}{2 k} & \frac{P^{2}}{2 k}
\end{array}\right) .
$$

If we introduce the notation

$$
\mathcal{G}_{M}(z):=\operatorname{det}\left(h_{\alpha \bar{\beta}}\right)_{\alpha, \beta=1, \ldots, n}
$$

then we find

$$
\mathcal{G}_{\mathcal{D}_{1}^{J}}(z, w)=\frac{2 k \mu}{(1-w \bar{w})^{3}}, \quad z \in \mathbb{C}, \quad|w|<1 .
$$

\section{Elements of geometry of the Siegel-Jacobi ball}

\subsection{The balanced metric}

The Jacobi algebra is the semi-direct $\operatorname{sum} \mathfrak{g}_{n}^{J}:=\mathfrak{h}_{n} \rtimes \mathfrak{s p}(n, \mathbb{R})_{\mathbb{C}}[6,7,9]$. The Heisenberg algebra $\mathfrak{h}_{n}$ is generated by the boson creation (respectively, annihilation) operators $a_{i}^{\dagger}\left(a_{i}\right), i, j=1, \ldots, n$, which verify the canonical commutation relations

$$
\left[a_{i}, a_{j}^{\dagger}\right]=\delta_{i j}, \quad\left[a_{i}, a_{j}\right]=\left[a_{i}^{\dagger}, a_{j}^{\dagger}\right]=0 .
$$

$\mathfrak{h}_{n}$ is an ideal in $\mathfrak{g}_{n}^{J}$, i.e., $\left[\mathfrak{h}_{n}, \mathfrak{g}_{n}^{J}\right]=\mathfrak{h}_{n}$, determined by the commutation relations:

$$
\begin{aligned}
& {\left[a_{k}^{\dagger}, K_{i j}^{+}\right]=\left[a_{k}, K_{i j}^{-}\right]=0,} \\
& {\left[a_{i}, K_{k j}^{+}\right]=\frac{1}{2} \delta_{i k} a_{j}^{\dagger}+\frac{1}{2} \delta_{i j} a_{k}^{\dagger}, \quad\left[K_{k j}^{-}, a_{i}^{\dagger}\right]=\frac{1}{2} \delta_{i k} a_{j}+\frac{1}{2} \delta_{i j} a_{k},} \\
& {\left[K_{i j}^{0}, a_{k}^{\dagger}\right]=\frac{1}{2} \delta_{j k} a_{i}^{\dagger}, \quad\left[a_{k}, K_{i j}^{0}\right]=\frac{1}{2} \delta_{i k} a_{j} .}
\end{aligned}
$$

$K_{i j}^{ \pm, 0}$ are the generators of the $\mathfrak{s p}(\mathbb{R})_{\mathbb{C}}$ algebra:

$$
\begin{aligned}
& {\left[K_{i j}^{-}, K_{k l}^{-}\right]=\left[K_{i j}^{+}, K_{k l}^{+}\right]=0, \quad 2\left[K_{i j}^{-}, K_{k l}^{0}\right]=K_{i l}^{-} \delta_{k j}+K_{j l}^{-} \delta_{k i},} \\
& 2\left[K_{i j}^{-}, K_{k l}^{+}\right]=K_{k j}^{0} \delta_{l i}+K_{l j}^{0} \delta_{k i}+K_{k i}^{0} \delta_{l j}+K_{l i}^{0} \delta_{k j}, \\
& 2\left[K_{i j}^{+}, K_{k l}^{0}\right]=-K_{i k}^{+} \delta_{j l}-K_{j k}^{+} \delta_{l i}, \quad 2\left[K_{j i}^{0}, K_{k l}^{0}\right]=K_{j l}^{0} \delta_{k i}-K_{k i}^{0} \delta_{l j} .
\end{aligned}
$$

Applying the same arguments as in the proof of Remark 3 in [10] (see also the Appendix in [10], where the notion of homogeneous reductive space in the meaning of Nomizu [63] is recalled; also see [11]) to the Jacobi algebra $\mathfrak{g}_{n}^{J}$ determined by (3.1), (3.2), (3.3), we make the

Remark 3.1. The Jacobi group $G_{n}^{J}$ is an unimodular, non-reductive, algebraic group of HarishChandra type. The Siegel-Jacobi domain $\mathcal{D}_{n}^{J}$ is a reductive, non-symmetric manifold associated to the Jacobi group $G_{n}^{J}$ by the generalized Harish-Chandra embedding.

We have attached to the Jacobi group $G_{n}^{J}:=\mathrm{H}_{n} \rtimes \mathrm{Sp}(n, \mathbb{R})_{\mathbb{C}}$ coherent states based on the Siegel-Jacobi ball $\mathcal{D}_{n}^{J}$ [7]. Perelomov's CS vectors [64] associated to the group $G_{n}^{J}$ with Lie algebra the Jacobi algebra $\mathfrak{g}_{n}^{J}$ based on the complex $d$-dimensional $(d=n(n+3) / 2)$ manifold the Siegel-Jacobi ball - $\mathcal{D}_{n}^{J} \approx \mathrm{H}_{n} / \mathbb{R} \times \mathrm{Sp}(n, \mathbb{R})_{\mathbb{C}} / \mathrm{U}(n)=\mathbb{C}^{n} \times \mathcal{D}_{n}$ - have been defined as [7]

$$
e_{z, W}=\exp (\boldsymbol{X}) e_{0}, \quad \boldsymbol{X}:=\sqrt{\mu} \sum_{i=1}^{n} z_{i} \boldsymbol{a}_{i}^{\dagger}+\sum_{i, j=1}^{n} w_{i j} \boldsymbol{K}_{i j}^{+}, \quad z \in \mathbb{C}^{n}, \quad W \in \mathcal{D}_{n}
$$


The vector $e_{0}$ appearing in (3.4) verifies the relations

$$
\begin{aligned}
& \boldsymbol{a}_{i} e_{0}=0, \quad i=1, \ldots, n, \\
& \boldsymbol{K}_{i j}^{+} e_{0} \neq 0, \quad \boldsymbol{K}_{i j}^{-} e_{0}=0, \quad \boldsymbol{K}_{i j}^{0} e_{0}=\frac{k}{4} \delta_{i j} e_{0}, \quad i, j=1, \ldots, n .
\end{aligned}
$$

In (3.5) $e_{0}=e_{0}^{H} \otimes e_{0}^{K}$, where $e_{0}^{H}$ is the minimum weight vector (vacuum) for the Heisenberg group $\mathrm{H}_{n}$, while $e_{0}^{K}$ is the extremal weight vector for $\operatorname{Sp}(n, \mathbb{R})_{\mathbb{C}}$ corresponding to the weight $k$ in (3.5), and $\mu$ parametrizes the Heisenberg group [7, 9, 15]. Holomorphic irreducible representations of the Jacobi group based on Siegel-Jacobi domains have been studied in mathematics $[22,23,72,73,74]$. In $[5,9,15]$ we have compared our results on the geometry of $\mathcal{D}_{n}^{J}$ and holomorphic representations of $G_{n}^{J}$ obtained using CS with those of the mentioned mathematicians.

The scalar product $K: \mathcal{D}_{n}^{J} \times \mathcal{D}_{n}^{J} \rightarrow \mathbb{C}, K(\bar{x}, \bar{V} ; y, W)=\left(e_{x, V}, e_{y, W}\right)_{k \mu}$ is $[7,15]$ :

$$
\begin{aligned}
& \left(e_{x, V}, e_{y, W}\right)_{k \mu}=\operatorname{det}(U)^{k / 2} \exp \mu F(\bar{x}, \bar{V} ; y, W), \quad U=\left(\mathbb{1}_{n}-W \bar{V}\right)^{-1}, \\
& 2 F(\bar{x}, \bar{V} ; y, W)=2(x, U y)+(V \bar{y}, U y)+(x, U W \bar{x}) .
\end{aligned}
$$

If we denote $\zeta=(x, V), \zeta^{\prime}=(y, W)$, then we find for the normalized Bergman kernel (2.10) of the Siegel-Jacobi ball the expression

$$
\kappa_{\mathcal{D}_{n}^{J}}\left(\zeta, \zeta^{\prime}\right)=\kappa_{\mathcal{D}_{n}}(V, W) \exp \mu\left[2 F\left(\zeta, \zeta^{\prime}\right)-F(\zeta)-F\left(\zeta^{\prime}\right)\right],
$$

where $\kappa_{\mathcal{D}_{n}}(V, W)$ is the normalized Bergman kernel of the Siegel ball

$$
\kappa_{\mathcal{D}_{n}}(V, W)=\operatorname{det}^{k / 2}\left[\frac{\left(\mathbb{1}_{n}-V \bar{V}\right)\left(\mathbb{1}_{n}-W \bar{W}\right)}{\left(\mathbb{1}_{n}-W \bar{V}\right)^{2}}\right] .
$$

We find for the Calabi diastasis (2.11) of the Siegel-Jacobi ball the expression

$$
\begin{aligned}
& D_{\mathcal{D}_{n}^{J}}\left(\zeta, \zeta^{\prime}\right)=D_{\mathcal{D}_{n}}(V, W)+2 \mu\left[\Re F(\zeta)+\Re F\left(\zeta^{\prime}\right)-2 \Re F\left(\zeta, \zeta^{\prime}\right)\right], \\
& D_{\mathcal{D}_{n}}(V, W)=-2 \ln \left|\kappa_{\mathcal{D}_{n}}(V, W)\right| .
\end{aligned}
$$

In particular, the reproducing kernel $K(z, W)=\left(e_{z, W}, e_{z, W}\right)_{k \mu}, z \in \mathbb{C}^{n}, W \in \mathcal{D}_{n}$ is

$$
\begin{aligned}
& K(z, W)=\operatorname{det}(M)^{k / 2} \exp \mu F, \quad M=\left(\mathbb{1}_{n}-W \bar{W}\right)^{-1}, \\
& 2 F=2 \bar{z}^{t} M z+z^{t} \bar{W} M z+\bar{z}^{t} M W \bar{z}, \quad 2 F=2 \bar{\eta}^{t} \eta-\eta^{t} \bar{W} \eta-\bar{\eta}^{t} W \bar{\eta}, \\
& \eta=M(z+W \bar{z}), \quad z=\eta-W \bar{\eta} .
\end{aligned}
$$

The Hilbert space of holomorphic functions $\mathcal{F}_{K}$ associated to the kernel $K$ given by (3.9) is endowed with the scalar product of the type (2.3)

$$
\begin{aligned}
& (\phi, \psi)_{\mathcal{F}_{K}}=\Lambda_{n} \int_{z \in \mathbb{C}^{n} ; W=W^{t} ; 1-W \bar{W}>0} \bar{f}_{\phi}(z, W) f_{\psi}(z, W) Q K^{-1} \mathrm{~d} z \mathrm{~d} W, \\
& \mathrm{~d} z=\prod_{i=1}^{n} \mathrm{~d} \Re z_{i} \mathrm{~d} \Im z_{i}, \quad \mathrm{~d} W=\prod_{1 \leq i \leq j \leq n} \mathrm{~d} \Re w_{i j} \mathrm{~d} \Im w_{i j},
\end{aligned}
$$

where the normalization constant $\Lambda_{n}$ is given by

$$
\Lambda_{n}=\mu^{n} \frac{k-3}{2 \pi^{n(n+3) / 2}} \prod_{i=1}^{n-1} \frac{\left(\frac{k-3}{2}-n+i\right) \Gamma(k+i-2)}{\Gamma[k+2(i-n-1)]},
$$


and the density of volume is [7] (see also Lemma A.2)

$$
Q(z, W)=\operatorname{det}\left(\mathbb{1}_{n}-W \bar{W}\right)^{-(n+2)} .
$$

The manifold $\mathcal{D}_{n}^{J}$ has the Kähler potential (3.12), $f=\log K$, with $K$ given by (3.9),

$$
\begin{aligned}
f= & -\frac{k}{2} \log \operatorname{det}\left(\mathbb{1}_{n}-W \bar{W}\right) \\
& +\mu\left\{\bar{z}^{t}\left(\mathbb{1}_{n}-W \bar{W}\right)^{-1} z+\frac{1}{2} z^{t}\left[\bar{W}\left(\mathbb{1}_{n}-W \bar{W}\right)^{-1}\right] z+\frac{1}{2} \bar{z}^{t}\left[\left(\mathbb{1}_{n}-W \bar{W}\right)^{-1} W\right] \bar{z}\right\} .
\end{aligned}
$$

Because the symmetry of the matrix $W \in \mathcal{D}_{n}$, we write down the Kähler two-form on the Siegel-Jacobi ball as

$$
\begin{aligned}
-\mathrm{i} \omega_{\mathcal{D}_{n}^{J}}(z, W)= & h_{i \bar{j}} \mathrm{~d} z_{i} \wedge \mathrm{d} \bar{z}_{j}+\sum_{p \leq q}\left(h_{i \bar{p} \bar{q}} \mathrm{~d} z_{i} \wedge \mathrm{d} \bar{w}_{p q}-\bar{h}_{i \bar{p} \bar{q}} \mathrm{~d} \bar{z}_{i} \wedge \mathrm{d} w_{p q}\right) \\
& +\sum_{p \leq q ; m \leq n} h_{p q \bar{m} \bar{n}} \mathrm{~d} w_{p q} \wedge \mathrm{d} \bar{w}_{m n} .
\end{aligned}
$$

We use the following notation for the matrix of the metric

$$
\begin{aligned}
& h=\left(\begin{array}{ll}
h_{1} & h_{2} \\
h_{3} & h_{4}
\end{array}\right)=\left(\begin{array}{cc}
h_{i \bar{j}} & h_{i \bar{p} \bar{q}} \\
h_{p q \bar{i}} & h_{p q \bar{m} \bar{n}}
\end{array}\right) \in M(n(n+3) / 2, \mathbb{C}), \\
& p \leq q, \quad m \leq n, \quad h=h^{*},
\end{aligned}
$$

where $h_{1} \in M(n, \mathbb{C}), h_{2} \in M(n \times n(n+1) / 2, \mathbb{C}), h_{3} \in M(n(n+1) / 2 \times n, \mathbb{C}), h_{4} \in M(n(n+$ $1) / 2, \mathbb{C})$.

We use the following convention: indices of $z \in M(n \times 1, \mathbb{C})$ are denoted with $i, j, k, l$; indices of $W=W^{t}, W \in M(n, \mathbb{C})$ are denoted with $m, n, p, q, r, s, t, u, v$.

Let as write the "inverse" $h^{-1}$ of the matrix $h(3.14)$ as

$$
h^{-1}=\left(\begin{array}{cc}
h^{1} & h^{2} \\
h^{3} & h^{4}
\end{array}\right)=\left(\begin{array}{cc}
\left(h^{1}\right)_{i \bar{j}} & \left(h^{2}\right)_{i \bar{p} \bar{q}} \\
\left(h^{3}\right)_{p q \bar{i}} & \left(h^{4}\right)_{p q \bar{m} \bar{n}}
\end{array}\right), \quad p \leq q, \quad m \leq n,
$$

where the matrices $h^{1}, h^{2}, h^{3}, h^{4}$ have the same dimensions as the matrices $h_{1}, h_{2}, h_{3}$, respectively $h_{4}$.

In fact, we shall determine the "inverse" (3.15) of (3.14) such that

$$
\left(\begin{array}{ll}
h_{1} & h_{2} \\
h_{3} & h_{4}
\end{array}\right)\left(\begin{array}{ll}
h^{1} & h^{2} \\
h^{3} & h^{4}
\end{array}\right)=\left(\begin{array}{cc}
\mathbb{1}_{n} & 0 \\
0 & \Delta
\end{array}\right)
$$

where $\Delta$ is defined in (2.26).

It is useful to introduce the notation

$$
f_{p q}:=1-\frac{1}{2} \delta_{p q}
$$

We shall prove the following (partial results have been presented in [6, Proposition 11], [7, Proposition 3.11] and [9, Proposition 1]):

Theorem 3.2. The Kähler two-form $\omega_{\mathcal{D}_{n}^{J}}$, associated with the balanced metric of the SiegelJacobi ball $\mathcal{D}_{n}^{J}, G_{n}^{J}$-invariant to the action (2.16) is

$$
\begin{aligned}
& -\mathrm{i} \omega_{\mathcal{D}_{n}^{J}}(z, W)=\frac{k}{2} \operatorname{Tr}(\mathcal{B} \wedge \overline{\mathcal{B}})+\mu \operatorname{Tr}\left(\mathcal{A}^{t} \bar{M} \wedge \overline{\mathcal{A}}\right), \quad \mathcal{A}=\mathrm{d} z+\mathrm{d} W \bar{\eta}, \\
& \mathcal{B}=M \mathrm{~d} W, \quad M=\left(\mathbb{1}_{n}-W \bar{W}\right)^{-1} .
\end{aligned}
$$


The matrix (3.14) of the metric on $\mathcal{D}_{n}^{J}$ has the matrix elements (3.18):

$$
\begin{aligned}
h_{i \bar{j}}=\mu & \bar{M}_{i j}, \\
h_{i \bar{p} \bar{q}}= & \mu\left(\eta_{q} \bar{M}_{i p}+\eta_{p} \bar{M}_{i q}\right) f_{p q}, \\
h_{p q \bar{i}}= & \mu\left(\bar{\eta}_{q} \bar{M}_{p i}+\bar{\eta}_{p} \bar{M}_{q i}\right) f_{p q}, \\
h_{p q \bar{m} \bar{n}}= & \frac{k}{2} h_{p q \bar{m} \bar{n}}^{k}+\mu h_{p q \bar{m} \bar{n}}^{\mu}, \\
h_{p q \bar{m} \bar{n}}^{k}= & 2\left(M_{m p} M_{n q}+M_{m q} M_{n p}\right)-2 M_{m p}\left(M_{n p} \delta_{p q}+M_{m q} \delta_{m n}\right)+M_{m p}^{2} \delta_{p q} \delta_{m n} \\
= & 2 M_{m p} M_{n q}\left(1-\delta_{p q}\right)+2 M_{m q} M_{n p}\left(1-\delta_{m n}\right)+M_{m p}^{2} \delta_{p q} \delta_{m n}, \\
h_{p q \bar{m} \bar{n}}^{\mu}= & \bar{\eta}_{p}\left(\eta_{n} M_{m q}+\eta_{m} M_{n q}\right)+\bar{\eta}_{q}\left(\eta_{n} M_{m p}+\eta_{m} M_{n p}\right)-\bar{\eta}_{p}\left(\eta_{n} M_{m p}\right. \\
& \left.+\eta_{m} M_{n p}\right) \delta_{p q}-\eta_{m}\left(\bar{\eta}_{p} M_{m q}+\bar{\eta}_{q} M_{m p}\right) \delta_{m n}+\bar{\eta}_{p} \eta_{m} M_{m p} \delta_{p q} \delta_{m n} \\
= & {\left[\bar{\eta}_{p}\left(\eta_{n} \bar{M}_{q m}+\eta_{m} \bar{M}_{q n}\right)+\bar{\eta}_{q}\left(\eta_{n} \bar{M}_{p m}+\eta_{m} \bar{M}_{p n}\right)\right] f_{p q} f_{m n} . }
\end{aligned}
$$

The "inverse" $h^{-1}$ of the metric matrix $h$ which verif ies (3.16), with components (3.18), obtained with the inverse (2.27) of $h^{k}(3.18 \mathrm{e})$ has the elements $h^{1}-h^{4}$ given by

$$
\begin{aligned}
& \left(h^{1}\right)_{i j}=\theta \bar{M}_{i j}^{-1}, \quad \theta=\frac{1}{\mu}+\alpha \frac{2}{k}, \quad \alpha=\eta^{t} \bar{M}^{-1} \bar{\eta}=\bar{\eta}_{n} S_{n}, \quad S_{n}=\sum \eta_{q} \bar{M}_{q n}^{-1}, \\
& \left(h^{2}\right)_{i \bar{m} \bar{n}}=-\frac{1}{k}\left(S_{n} \bar{M}_{i m}^{-1}+S_{m} \bar{M}_{i n}^{-1}\right), \\
& \left(h^{3}\right)_{m n \bar{i}}=-\frac{1}{k}\left(\bar{S}_{n} \bar{M}_{m i}^{-1}+\bar{S}_{m} \bar{M}_{n i}^{-1}\right), \\
& \left(h^{4}\right)_{p q \bar{m} \bar{n}}=\frac{2}{k}\left(h^{k}\right)_{p q \bar{m} \bar{n}}^{-1}=\frac{1}{k}\left(\bar{M}_{q n}^{-1} \bar{M}_{p m}^{-1}+\bar{M}_{p n}^{-1} \bar{M}_{q m}^{-1}\right) .
\end{aligned}
$$

The determinant of the metric matrix $h$ is

$$
\mathcal{S}_{\mathcal{D}_{n}^{J}}(z, W)=\operatorname{det} h_{\mathcal{D}_{n}^{J}}(z, W)=\left(\frac{k}{2}\right)^{\frac{n(n+1)}{2}} \mu^{n} \operatorname{det}\left(\mathbb{1}_{n}-W \bar{W}\right)^{-(n+2)} .
$$

In the case $n=1$ formulas (3.18), (3.19), (3.20) became the formulas (2.35), (2.36), respectively (2.37), with $2 k \leftrightarrow \frac{k}{2}$.

Proof. Firstly, we determine the matrix elements of the metric $h$ on the Siegel-Jacobi disk $\mathcal{D}_{n}^{J}$ applying formula (2.1) to the Kähler potential (3.12) as in (3.13).

We use the formulas (see [9, equations (4.23)])

$$
\begin{aligned}
& \frac{\partial M_{a b}}{\partial w_{i k}}=M_{a i} X_{k b}+M_{a k} X_{i b}-M_{a i} X_{i b} \delta_{i k}, \quad \text { where } \quad X=X^{t}=\bar{W} M=\bar{M} \bar{W}, \\
& \frac{\partial X_{a b}}{\partial w_{i k}}=X_{a i} X_{b k}+X_{a k} X_{i b}-X_{a i} X_{i b} \delta_{i k}, \\
& \frac{\partial \bar{X}_{a b}}{\partial w_{i k}}=M_{a i} M_{b k}+M_{a k} M_{b i}-M_{a i} M_{b k} \delta_{i k} .
\end{aligned}
$$

From (3.10), we get

$$
\begin{aligned}
& \frac{\partial \eta_{q}}{\partial z_{l}}=M_{q l}, \\
& \frac{\partial \bar{\eta}_{q}}{\partial z_{j}}=X_{q j}, \\
& \frac{\partial \eta_{t}}{\partial w_{p q}}=M_{t p} \bar{\eta}_{q}+M_{t q} \bar{\eta}_{p}-M_{t p} \bar{\eta}_{p} \delta_{p q},
\end{aligned}
$$




$$
\frac{\partial \bar{\eta}_{n}}{\partial w_{p q}}=\bar{\eta}_{p} X_{q n}+\bar{\eta}_{q} X_{p n}-\bar{\eta}_{q} X_{p n} \delta_{p q}
$$

With (2.26), (3.21) and (3.22), we find the matrix elements (3.18) of the metric of the SiegelJacobi ball.

Now we prove (3.18e). We calculate

$$
h_{m n \bar{p} \bar{q}}^{k}=\frac{\partial}{\partial \bar{w}_{p q}} J_{m n}, \quad J_{m n}=\frac{\partial}{\partial w_{m n}} \ln \operatorname{det} M .
$$

With formula

$$
\frac{\mathrm{d}(\operatorname{det} A)}{\mathrm{d} t}=\operatorname{det} A \operatorname{Tr}\left(A^{-1} \frac{\partial A}{\partial t}\right),
$$

we have

$$
J_{m n}=M_{i j}^{-1} \frac{\partial M_{j i}}{\partial w_{m n}} .
$$

We find

$$
J_{m n}=X_{n m}+X_{m n}-X_{m n} \delta_{m n}=2 X_{m n} f_{m n} .
$$

In order to obtain $h_{m n \bar{p} \bar{q}}^{k}$, we calculate

$$
\frac{\partial X_{n m}}{\partial \bar{w}_{p q}}=\frac{\partial \bar{w}_{n k}}{\partial \bar{w}_{p q}} M_{k n}+w_{n k} \frac{\partial M_{k b}}{\partial \bar{w}_{p q}} .
$$

But with

$$
\frac{\partial M_{k m}}{\partial \bar{w}_{p q}}=\overline{\left(\frac{M_{m k}}{\partial w_{p q}}\right)}, \quad \text { and with } \quad \mathbb{1}_{n}+\bar{W} \bar{X}=\bar{M},
$$

we find

$$
\frac{\partial X_{n m}}{\partial \bar{w}_{p q}}=2\left(M_{p m} \bar{M}_{n q}+M_{q m} \bar{M}_{n p}-M_{p m} \bar{M}_{n q} \delta_{p q}\right)-\left(2 M_{p m} \bar{M}_{m q}-M_{p m} \bar{M}_{m q} \delta_{p q}\right) \delta_{m n},
$$

i.e., we reobtain formula (3.18e).

With (3.18e), we have

$$
\begin{aligned}
& h_{p p \bar{m} \bar{m}}^{k}=M_{m p}^{2}, \\
& h_{p p \bar{m} \bar{n}}^{k}=2 M_{m p} M_{n q}, \quad m<n, \\
& h_{p q \bar{m} \bar{m}}^{k}=2 M_{m p} M_{m q}, \quad p<q, \\
& h_{p q \bar{m} \bar{n}}^{k}=2\left(M_{m p} M_{n q}+M_{m q} M_{n p}\right), \quad p<q, \quad m<n .
\end{aligned}
$$

In order to find $-\mathrm{i} \frac{2}{k} \mathrm{~d} \omega_{\mathcal{D}_{n}}(W)$, we make the summation

$$
\begin{aligned}
\sum h_{p p \bar{m} \bar{m}}^{k} \mathrm{~d} w_{p p} \wedge \mathrm{d} \bar{w}_{m m}+\sum_{m<n} h_{p p \bar{m} \bar{n}}^{k} \mathrm{~d} w_{p p} \wedge \mathrm{d} \bar{w}_{m n} \\
\quad+\sum_{p<q} h_{p q \bar{m} \bar{m}}^{k} \mathrm{~d} w_{p q} \wedge \mathrm{d} \bar{w}_{m m}+\sum_{p<q, m<n} h_{p q \bar{m} \bar{n}}^{k} \mathrm{~d} w_{p q} \wedge \mathrm{d} \bar{w}_{m n} \\
=\sum M_{m p} \bar{M}_{p m} \mathrm{~d} w_{p p} \wedge \mathrm{d} \bar{w}_{m m}+2 \sum_{m<n} M_{m p} M_{p n} \mathrm{~d} w_{p p} \wedge \mathrm{d} \bar{w}_{m n}
\end{aligned}
$$




$$
\begin{aligned}
& +2 \sum_{p<q} M_{m p} M_{m q}+2 \sum_{p<q, m<n}\left(M_{m p} M_{n q}+M_{m q} M_{n p}\right) \mathrm{d} w_{p q} \wedge \mathrm{d} \bar{w}_{m n} \\
= & \sum M_{m p} \bar{M}_{p m} \mathrm{~d} w_{p p} \wedge \mathrm{d} \bar{w}_{m m}+\sum_{m \neq n} M_{m p} M_{p n} \mathrm{~d} w_{p p} \wedge \mathrm{d} \bar{w}_{m n} \\
& +\sum_{p \neq q} M_{m p} M_{n q} \mathrm{~d} w_{p q} \wedge \mathrm{d} \bar{w}_{m m}+\sum_{p \neq q, m \neq n} M_{m p} M_{n q} \mathrm{~d} w_{p q} \wedge \mathrm{d} \bar{w}_{m n} \\
= & (M \mathrm{~d} W)_{m q} \wedge(\bar{M} \mathrm{~d} \bar{W})_{q m} .
\end{aligned}
$$

We write down (3.25) as

$$
-\mathrm{i} \frac{2}{k} \mathrm{~d} \omega_{\mathcal{D}_{n}}(W)=\operatorname{Tr}(M \mathrm{~d} W \wedge \bar{M} \mathrm{~d} \bar{W}) .
$$

Now we make the summation

$$
\begin{aligned}
\sum_{p \leq q} h_{i \bar{p} \bar{q}}^{\mu} \mathrm{d} z_{i} \wedge \mathrm{d} \bar{w}_{p q} & =\sum h_{i \bar{p} \bar{p}}^{\mu} \mathrm{d} z_{i} \wedge \mathrm{d} \bar{w}_{p p}+\sum_{p<q} h_{i \bar{p} \bar{q}}^{\mu} \mathrm{d} z_{i} \wedge \mathrm{d} \bar{w}_{p q} \\
& =\bar{M}_{i p} \eta_{p} \mathrm{~d} z_{i} \wedge \mathrm{d} \bar{w}_{p p}+\sum_{p<q}\left(\bar{M}_{i p} \eta_{q}+\bar{M}_{i q} \eta_{p}\right) \mathrm{d} z_{i} \wedge \mathrm{d} \bar{w}_{p q} \\
& =(M \mathrm{~d} z)_{p} \wedge(\mathrm{d} \bar{W} \eta)_{p} .
\end{aligned}
$$

We calculate the sum

$$
H^{\mu}:=\sum_{p \leq q, m \leq n} h_{p q \bar{m} \bar{n}}^{\mu} \mathrm{d} w_{p q} \wedge \mathrm{d} \bar{w}_{m n} .
$$

We have

$$
\begin{aligned}
H^{\mu}= & \sum h_{p p \bar{m} \bar{m}}^{\mu} \mathrm{d} w_{p p} \wedge \bar{w}_{m m}+\sum_{m<n} h_{p p \bar{m} \bar{n}}^{\mu} \mathrm{d} w_{p p} \wedge \bar{w}_{m n} \\
& +\sum_{p<q} h_{p q \bar{m} \bar{m}}^{\mu} \mathrm{d} w_{p q} \wedge \bar{w}_{m m}+\sum_{p<q, m<n} h_{p q \bar{m} \bar{n}}^{\mu} \mathrm{d} w_{p q} \wedge \bar{w}_{m n},
\end{aligned}
$$

and finally we get

$$
H^{\mu}=M_{m q} \mathrm{~d} w_{q p} \wedge \mathrm{d} \bar{w}_{m n} \eta_{n}=(M \mathrm{~d} W \bar{\eta})_{m} \wedge(\mathrm{d} \bar{W} \eta)_{m} .
$$

Putting together (3.26), (3.25) and (3.27), we have proved (3.17).

In order to check out the homogeneity of the Kähler two-form $\omega_{\mathcal{D}_{n}^{J}}(3.17)$, we use the formulas

$$
\begin{aligned}
& \mathrm{d} W_{1}=\left(W q^{*}+p^{*}\right)^{-1} \mathrm{~d} W(\bar{q} W+\bar{p})^{-1}, \\
& M_{1}=(q \bar{W}+p) M\left(W q^{*}+p^{*}\right), \\
& \mathrm{d} z_{1}=\left(W q^{*}+p^{*}\right)^{-1}(\mathrm{~d} W V+\mathrm{d} z), \quad V=-q^{*}\left(W q^{*}+p^{*}\right)^{-1}[\eta+\alpha-W(\bar{\eta}+\bar{\alpha})]-\bar{\alpha}, \\
& \eta_{1}=p(\alpha+\eta)+q(\bar{\alpha}+\bar{\eta}), \quad \mathcal{A}_{1}=\left(W q^{*}+p^{*}\right)^{-1} \mathcal{A} .
\end{aligned}
$$

Now we calculate the "inverse" (3.15) $h^{-1}$ of the matrix $h$ whose elements are given by (3.18).

We have (see, e.g., in [56, (1) and (2), p. 30]): if the matrices $h_{1}, h_{4}-h_{3} h_{1}^{-1} h_{2}, h_{4}$ and respectively $h_{1}-h_{2} h_{4}^{-1} h_{3}$ are nonsingular, then

$$
\begin{aligned}
& h^{1}=\left(h_{1}-h_{2} h_{4}^{-1} h_{3}\right)^{-1}=h_{1}^{-1}+h_{1}^{-1} h_{2} h^{4} h_{3} h_{1}^{-1}, \\
& h^{2}=-h_{1}^{-1} h_{2} h^{4},
\end{aligned}
$$




$$
\begin{aligned}
& h^{3}=-h^{4} h_{3} h_{1}^{-1}, \\
& h^{4}=\left(h_{4}-h_{3} h_{1}^{-1} h_{2}\right)^{-1} .
\end{aligned}
$$

In order to calculate $h^{4}$, we write $(3.30 \mathrm{~d})$ as

$$
\left(h^{4}\right)^{-1}=h_{4}-\mu h^{I}, \quad \text { where } \quad h^{I}:=\frac{1}{\mu} h_{3} h_{1}^{-1} h_{2} .
$$

With formulas (3.18a)-(3.18c), we get

$$
\begin{aligned}
\left(h^{I}\right)_{p q \bar{m} \bar{n}} & =h_{p q \bar{i}}\left(h_{1}\right)_{i j}^{-1} h_{j \bar{m} \bar{n}}=\left(\bar{\eta}_{q} \bar{M}_{p i}+\bar{\eta}_{p} \bar{M}_{q i}\right) \bar{M}_{i j}^{-1}\left(\eta_{n} \bar{M}_{j m}+\eta_{m} \bar{M}_{j n}\right) f_{p q} f_{m n} \\
& =\left(\bar{\eta}_{q} \bar{M}_{p i}+\bar{\eta}_{p} \bar{M}_{q i}\right)\left(\eta_{n} \delta_{i m}+\eta_{m} \delta_{i m}\right) f_{p q} f_{m n} \\
& =\left[\bar{\eta}_{q}\left(\eta_{n} \bar{M}_{p m}+\eta_{m} \bar{M}_{p n}\right)+\bar{\eta}_{p}\left(\eta_{n} \bar{M}_{q m}+\eta_{m} \bar{M}_{q n}\right)\right] f_{p q} f_{m n},
\end{aligned}
$$

and with equation (3.18f) we get

$$
h^{I}=h^{\mu} .
$$

With (3.31), we obtain

$$
\left(h^{4}\right)^{-1}=\frac{k}{2} h^{k}
$$

and $(3.19 \mathrm{~d})$, where $h^{k}$ has the expression (3.18e), while $\left(h^{k}\right)^{-1}$ has the expression $(2.27)$.

In order to calculate $h^{2}$, taking into account (3.32), we write (3.30b) as

$$
h^{2}=-\left(h_{1}\right)^{-1} h^{J}, \quad \text { where } \quad h^{J}:=\frac{2}{k} h_{2}\left(h^{k}\right)^{-1} .
$$

With formulas (3.18b), (B.11), we have

$$
\begin{aligned}
\left(h^{J}\right)_{j \bar{m} \bar{n}}= & 2 \frac{\mu}{k} \sum_{p \leq q} f_{p q}\left(\eta_{q} \bar{M}_{j p}+\eta_{p} \bar{M}_{j q}\right) k_{p q \bar{m} \bar{n}} \\
= & 2 \frac{\mu}{k}\left[\sum_{p} \eta_{p}\left(\mathbb{1}_{n}-W \bar{W}\right)_{p j}^{-1}\left(\mathbb{1}_{n}-W \bar{W}\right)_{n p}\left(\mathbb{1}_{n}-\bar{W} W\right)_{p m}\right] \\
& +\frac{\mu}{k} \sum_{p<q}\left[\eta_{q}\left(\mathbb{1}_{n}-W \bar{W}\right)_{p j}^{-1}+\eta_{p}\left(\mathbb{1}_{n}-W \bar{W}\right)_{p j}^{-1}\right] \\
& \times\left[\left(\mathbb{1}_{n}-W \bar{W}\right)_{n q}\left(\mathbb{1}_{n}-\bar{W} W\right)_{p m}+\left(\mathbb{1}_{n}-W \bar{w}\right)_{n p}\left(\mathbb{1}_{n}-\bar{W} W\right)_{q m}\right] \\
= & \frac{\mu}{k}\left[\sum _ { p \neq q } \left(\eta_{q}\left(\mathbb{1}_{n}-W \bar{W}\right)_{p j}^{-1}\left(\mathbb{1}_{n}-W \bar{W}\right)_{n q}\left(\mathbb{1}_{n}-\bar{W} W\right)_{p m}\right.\right. \\
& +\sum_{p \neq q} \eta_{p}\left(\mathbb{1}_{n}-W \bar{W}\right)_{q j}^{-1}\left(\mathbb{1}_{n}-W \bar{W}\right)_{n q}\left(\mathbb{1}_{n}-\bar{W} W\right)_{p m} \\
& \left.+2 \sum_{p} \eta_{p}\left(\mathbb{1}_{n}-W \bar{W}\right)_{p j}^{-1}\left(\mathbb{1}_{n}-W \bar{W}\right)_{n p}\left(\mathbb{1}_{n}-\bar{W} W\right)_{p m}\right] \\
& =\frac{\mu}{k}\left(S_{n} \delta_{j m}+S_{m} \delta_{j n}\right), \quad \text { where } \quad S_{n}:=\sum_{q} \eta_{q} \bar{M}_{q n}^{-1} .
\end{aligned}
$$

With (3.33), we get (3.19b) and similarly for (3.19c). 
In order to calculate $h^{1}$, we write (3.30a) as

$$
h^{1}=h_{1}^{-1}-h^{K} h_{1}^{-1}, \quad \text { where } \quad h^{K}:=h^{2} h_{3} .
$$

With formulas (3.19b) and (3.18c), we get

$$
\begin{aligned}
\left(h^{K}\right)_{i k} & =-\frac{\mu}{k} \sum_{m \leq n} f_{m n}\left(S_{n} \bar{M}_{i m}^{-1}+S_{m} \bar{M}_{i n}^{-1}\right)\left(\bar{\eta}_{n} \bar{M}_{m k}+\bar{\eta}_{m} \bar{M}_{n k}\right) \\
& =-\frac{\mu}{k}\left[\frac{1}{2} \sum_{m} 4 S_{m} \bar{\eta}_{m} \bar{M}_{i m}^{-1} \bar{M}_{m k}+\sum_{m<n}\left(S_{n} \bar{M}_{i m}^{-1}+S_{m} \bar{M}_{i n}^{-1}\right)\left(\bar{\eta}_{n} \bar{M}_{m k}+\bar{\eta}_{m} \bar{M}_{n k}\right)\right] \\
& =-\frac{2}{k} \mu \alpha \delta_{i k},
\end{aligned}
$$

where

$$
\alpha:=\eta^{t} \bar{M}^{-1} \bar{\eta}=\eta^{*} M^{-1} \eta=\bar{\eta}_{n} S_{n} .
$$

With (3.18a), we get (3.19a).

In order to prove (3.20), we use the relation

$$
\operatorname{det} h=\operatorname{det}\left(\begin{array}{ll}
h_{1} & h_{2} \\
h_{3} & h_{4}
\end{array}\right)=\operatorname{det} h_{1} \operatorname{det}\left(h_{4}-h_{3} h_{1}^{-1} h_{2}\right) .
$$

With (3.30d) and (3.32), we get

$$
\operatorname{det} h=\left(\frac{k}{2}\right)^{n} \operatorname{det} h_{1} \operatorname{det} h^{k} .
$$

(3.20) is obtained introducing in the above relation the expressions (3.18a) and (A.4).

Now we formulate several geometric properties of the Siegel-Jacobi ball relevant for the Berezin quantization of this manifold:

\section{Proposition 3.3.}

i) The homogeneous Kähler manifold $\mathcal{D}_{n}^{J}$ is contractible.

ii) The Kähler potential of the Siegel-Jacobi ball (3.12) is global. $\mathcal{D}_{n}^{J}$ is a Q.-K. Lu manifold, with normalized Bergman kernel (3.7) and nowhere vanishing diastasis (3.8).

iii) The manifold $\mathcal{D}_{n}^{J}$ is a quantizable manifold.

iv) $\mathcal{D}_{n}^{J}$ is projectively induced and the Jacobi group $G_{n}^{J}$ is a CS-type group.

v) $\mathcal{D}_{n}^{J}$ is a homogeneous reductive space.

Proof. $i$ ) We have proved in Theorem 3.2 (see also $[6,9]$ ) that $\mathcal{D}_{n}^{J}$ is a homogeneous Kähler manifold. We have shown in [9, Proposition 4.1] that the FC-transform FC: $(\eta, W) \rightarrow(z, W)$ expressed by (3.10) is a homogeneous Kähler diffeomorphism $\mathcal{D}_{n}^{J} \approx \mathcal{D}_{n} \times \mathbb{C}^{n}$, where the Siegel ball admits the realization (1.2). But $\mathcal{D}_{n}$ can be achieved as the open ball $\mathcal{B}_{n}$ (see, e.g., [62, p. 502])

$$
\mathcal{B}_{n}=\left\{W \in M(n, \mathbb{C}), \quad W=W^{t} \mid\|W\|<1\right\} .
$$

So, $\mathcal{D}_{n}^{J}$ is diffeomorphic with the product of the contractible spaces $\mathcal{D}_{n}$ and $\mathbb{C}^{n}$, and consequently, it is itself contractible. 
ii) We have for $(3.9) K(z, W)>0, \forall(z, W) \in \mathcal{D}_{n}^{J}$. The explicit expressions of the Kähler potential, normalized Bergman kernel and diastasis imply the assertions of $i i$ ), but they could be also derived from the Theorem 2.4, once we have proved $i$ ). Even more, Theorem 2.4 asserts that $\mathcal{D}_{n}^{J}$ admits a Berezin quantization.

iii), iv) We have observed in Theorem 3.2 that the Kähler two-form (3.17) is associated with the balanced metric on $\mathcal{D}_{n}^{J}$, and we apply Remark 2.3 .

$v$ ) This assertion was already mentioned in Remark 3.1, where it was used the explicit form (3.1), (3.2), (3.3) of the Jacobi algebra $\mathfrak{g}_{n}^{J}$. We mention it again here because in [13] (see Remark 1 there for a more precise formulation) we have proved that the CS-manifolds are reductive spaces.

The fact that the Jacobi algebra $\mathfrak{g}_{n}^{J}$ is a CS-algebra is well known, see, e.g., [50, Theorem 5.2] or [62, Example VII.2.3 , p. 294]. Using the explicit form of the base $\Phi(z, w)$ of orthonormal polynomials in which the Bergman kernel $K_{\mathcal{D}_{1}^{J}}(z, w)$ is developed [5], we have proved in [12, Proposition 2] that the Siegel-Jacobi disk is a CS-manifold. In [15] we have determined the base $\Phi(z, W)$ on $\mathcal{D}_{n}^{J}$ in which the reproducing kernel (3.6) admits a series expansion as in (2.6), but in Proposition 3.3 we have used Theorems 2.2, 2.4 and Remark 2.3 to prove directly Proposition 3.3.

\subsection{Ricci form and scalar curvature}

The Ricci form associated to the Kählerian two-form $\omega_{M}$ (1.3) is (see [61, p. 90])

$$
\rho_{M}(z):=\mathrm{i} \sum_{\alpha, \beta=1}^{n} \operatorname{Ric}_{\alpha \bar{\beta}}(z) \mathrm{d} z_{\alpha} \wedge \mathrm{d} \bar{z}_{\beta}, \quad \operatorname{Ric}_{\alpha \bar{\beta}}(z)=-\frac{\partial^{2}}{\partial z_{\alpha} \partial \bar{z}_{\beta}} \ln \mathcal{G}_{M}(z) .
$$

The scalar curvature at a point $p \in M$ of coordinates $z$ is (see [45, p. 294] or [41, p. 144])

$$
s_{M}(p):=\sum_{\alpha, \beta=1}^{n}\left(h_{\bar{\alpha} \beta}\right)^{-1} \operatorname{Ric}_{\alpha \bar{\beta}}(z) .
$$

The Bergman metric corresponds to the Kähler two-form (see references in [12])

$$
\omega_{M}^{1}=\mathrm{i} \partial \bar{\partial} \ln \mathcal{G} .
$$

Q.-K. $\mathrm{Lu}[55]$ has introduced for a bounded domain the positive definite $(1,1)$-form

$$
\tilde{\omega}_{M}(z):=\mathrm{i} \sum_{\alpha, \beta=1}^{n} \tilde{h}_{\alpha \bar{\beta}}(z) \mathrm{d} z_{\alpha} \wedge \mathrm{d} \bar{z}_{\beta}, \quad \tilde{h}_{\alpha \bar{\beta}}(z):=(n+1) h_{\alpha \bar{\beta}}(z)-\operatorname{Ric}_{\alpha, \beta}(z),
$$

which is Kähler, corresponding to the Kähler potential $\tilde{f}=\ln \left(K_{M}(z)^{n+1} \mathcal{G}(z)\right)$.

Now we prove

\section{Proposition 3.4.}

i) The Siegel-Jacobi ball $\mathcal{D}_{n}^{J}$ is not an Einstein manifold with respect to the homogeneous Kähler metric attached to the Kähler two-form (3.17), but it is one with respect to the Bergman metric corresponding to the Bergman Kähler two-form (3.36).

ii) The Ricci form (3.34) on the Siegel-Jacobi ball associated to the homogeneous Kählerian two-form (3.17) has the expression

$$
\rho_{\mathcal{D}_{n}^{J}}(z, W)=-\omega_{\mathcal{D}_{n}^{J}}^{1}(z, W)=-\mathrm{i}(n+2) \operatorname{Tr}(M \mathrm{~d} W \wedge \bar{M} \mathrm{~d} \bar{W}),
$$

while

$$
\rho_{\mathcal{D}_{n}}(W)=-\omega_{\mathcal{D}_{n}}^{1}(W)=-\mathrm{i}(n+1) \operatorname{Tr}(M \mathrm{~d} W \wedge \bar{M} \mathrm{~d} \bar{W}) .
$$


iii) The scalar curvature (3.35) is constant and negative

$$
s_{\mathcal{D}_{n}^{J}}(z, W)=-\frac{2}{k} n \frac{(n+1)(n+2)}{2} .
$$

iv) The Q.-K. Lu Kähler two-form (3.37) has the expression

$$
\tilde{\omega}_{\mathcal{D}_{n}^{J}}(z, W)=\frac{(n+1)(n+2)}{2} \omega_{\mathcal{D}_{n}^{J}}(z, W)-\rho_{\mathcal{D}_{n}^{J}}(W) .
$$

Proof. With formula (3.20) (or (3.11)) and (3.23), we find that the only nonzero components of the Ricci tensor are

$$
\left(\operatorname{Ric}_{m n \bar{p} \bar{q}}\right)_{\mathcal{D}_{n}^{J}}(z, W)=-(n+2) h_{m n \bar{p} \bar{q}}^{k}(W) .
$$

With calculation $(3.25)$, we find

$$
\rho_{\mathcal{D}_{n}^{J}}(z, W)=-\mathrm{i}(n+2) \bar{M}_{p i} \bar{M}_{j q} \mathrm{~d} w_{p j} \wedge \mathrm{d} \bar{w}_{q i} .
$$

Applying (3.35) for $\mathcal{D}_{n}^{J}$, with formulas (3.19d), (3.38), we get

$$
\begin{aligned}
s_{\mathcal{D}_{n}^{J}}(z, W) & :=\sum_{\alpha, \beta=1}^{n}(h)_{\bar{\alpha} \beta}^{-1} \operatorname{Ric}_{\alpha \bar{\beta}}(z, W)=\sum_{p \leq q ; m \leq n}(h)_{m n \bar{p} \bar{q}}^{-1}\left(\operatorname{Ric}_{p q \bar{m} \bar{n}}\right)_{\mathcal{D}_{n}^{J}} \\
& =-\frac{2}{k}(n+2) \sum_{p \leq q ; m \leq n}\left(h^{k}\right)_{m n \bar{p} \bar{q}}^{-1}\left(h^{k}\right)_{p q \bar{m} \bar{n}}=-\frac{2}{k}(n+2) \sum_{m \leq n} \Delta_{m n}^{m n} \\
& =-\frac{2}{k}(n+2) \frac{n(n+1)}{2} .
\end{aligned}
$$

The scalar curvature of the Siegel-Jacobi disk was calculated in [10, 12, 80].

\subsection{Laplace-Beltrami operator}

We introduce the formulas (3.19) into the expression (1.6) of the Laplace-Beltrami operator and we use Remark A.1. In the formula (3.40) of the Laplace-Beltrami operator on $\mathcal{D}_{n}^{J}$, we use the expression given in Proposition B.2 for the Laplace-Beltrami operator on $\mathcal{D}_{n}$. We introduce the notation (no summation!)

$$
\left(D_{z}\right)_{\mu \nu}=\left(e_{\mu \nu} \frac{\partial}{\partial z_{\mu \nu}}\right), \quad e_{\mu \nu}=\frac{1+\delta_{\mu \nu}}{2}, z_{\mu \nu}=z_{\nu \mu} .
$$

We obtain:

Proposition 3.5. The Laplace-Beltrami operator on the Siegel-Jacobi ball $\mathcal{D}_{n}^{J}$, invariant to the action (2.16) of the Jacobi group $G_{n}^{J}$, has the expression

$$
\begin{aligned}
& \Delta_{\mathcal{D}_{n}^{J}}(z, W)=\theta \frac{\partial}{\partial z^{t}} N \frac{\partial}{\partial \bar{z}}-\frac{2}{k}\left\{\operatorname{Tr}\left[S D_{W} N \frac{\partial}{\partial \bar{z}}\right]+c c\right\}+\frac{2}{k} \Delta_{\mathcal{D}_{n}}(W), \\
& N=\mathbb{1}_{n}-W \bar{W} .
\end{aligned}
$$

We have also the relations

$$
\Delta_{\mathcal{D}_{n}^{J}}(\ln \mathcal{G})=-s_{\mathcal{D}_{n}^{J}}=\frac{2}{k} n \frac{(n+1)(n+2)}{2} .
$$


Proof. We apply formula (1.6) with the matrix elements (3.19) and we use Remark A.1.

We recall that Theorem 2.5 in Berezin's paper [18] asserts essentially that

$$
\Delta_{M}(z)(\ln (\mathcal{G}(z)))=c t
$$

for the balanced metric plus other 3 conditions. The first equation in (3.41)

$$
s=-\Delta \ln \operatorname{det} h
$$

is a general recipe for calculation of scalar curvature, see, e.g., [41, equation (5.2.24), p. 253].

Also we recall that the Laplace operator on the Siegel-Jacobi ball was calculated in [79] in other coordinates. We have determined the Laplace-Beltrami operator on the Siegel-Jacobi disk in $[12]$.

\section{A Appendix}

\section{A.1 A remark}

Remark A.1. Let $\omega_{M}(z)$ be the non-degenerate two-form (1.3) on a complex manifold $M$, invariant to a invertible holomorphic transformation $z^{\prime}=z^{\prime}(z)$. Then the differential operator $\Delta_{M}$ given by (1.6) is also invariant to the transformation $z \rightarrow z^{\prime}$. In particular, $M$ may be a homogeneous Kähler manifold and $\Delta_{M}$ the corresponding Laplace-Beltrami operator.

Proof. Let us consider the action $G \times M \rightarrow M: g \times z=z^{\prime}$. Then we have

$$
-\mathrm{i} \omega_{M}\left(z^{\prime}\right)=h_{\lambda \bar{\gamma}}^{\prime}\left(z^{\prime}\right) \mathrm{d} z_{\lambda}^{\prime} \wedge \mathrm{d} \bar{z}_{\gamma}=h_{\lambda \bar{\gamma}}^{\prime}\left(z^{\prime}(z)\right) a_{\lambda \alpha} \bar{a}_{\gamma \beta} \mathrm{d} z_{\alpha} \wedge \mathrm{d} \bar{z}_{\beta}, \quad \text { where } \quad a_{\alpha \lambda}=\frac{\partial z_{\alpha}^{\prime}}{\partial z_{\lambda}} .
$$

If $\omega_{M}\left(z^{\prime}\right)=\omega_{M}(z)$, then

$$
h_{\alpha \bar{\beta}}(z)=h_{\lambda \gamma}^{\prime}\left(z^{\prime}(z)\right) a_{\lambda \alpha} \bar{a}_{\gamma \beta} .
$$

Now we consider the Laplace-Beltrami operator (1.6) in the point $z^{\prime}$. We have

$$
\frac{\partial}{\partial z_{\alpha}^{\prime}}=b_{\gamma \alpha} \frac{\partial}{\partial z_{\gamma}}, \quad \text { where } \quad b_{\lambda \beta}=\frac{\partial z_{\lambda}}{\partial z_{\beta}^{\prime}},
$$

and

$$
a_{\alpha \lambda} b_{\lambda \beta}=\delta_{\alpha \beta} .
$$

We have

$$
\Delta_{M}\left(z^{\prime}\right)=\left(h^{\prime}\left(z^{\prime}\right)\right)_{\alpha \bar{\beta}}^{-1} \bar{b}_{\gamma \alpha} b_{\rho \beta} \frac{\partial^{2}}{\partial \bar{z}_{\gamma} \partial z_{\rho}} .
$$

If $\Delta_{M}\left(z^{\prime}\right)=\Delta_{M}(z)$, then

$$
h_{\gamma \bar{\rho}}^{-1}(z)=\left(h^{\prime}\left(z^{\prime}(z)\right)\right)_{\alpha \bar{\beta}}^{-1} \bar{b}_{\gamma \alpha} b_{\rho \beta} .
$$

We write (A.1) as

$$
h=a^{t} h^{\prime} \bar{a},
$$

which implies

$$
h^{-1}=(\bar{a})^{-1}\left(h^{\prime}\right)^{-1}\left(a^{t}\right)^{-1}=\bar{b}\left(h^{\prime}\right)^{-1} b^{t},
$$

which is exactly (A.2). 


\section{A.2 A lemma}

Formula (3.20), modulo the numerical factor, was obtained in [7]. We recall the method to determine the Liouville form used in [7]. We have applied the following technique (see [38, Chapter IV]):

Lemma A.2. Let $z^{\prime}=f(g, z)$ denote the action of the group $G$ on the circular domain $M$. Let us determine the element $g \in G$ such that $z^{\prime}\left(z_{1}\right)=0$ Then the density of the volume form is $Q=|J|^{2}$, where $J$ is the Jacobian $J=\frac{\partial z^{\prime}}{\partial z}$.

The transformation with the desired properties is

$$
\begin{aligned}
z^{\prime}= & U\left(1-W_{1} \bar{W}_{1}\right)^{1 / 2}\left(1-W \bar{W}_{1}\right)^{-1} \\
& \times\left[z-\left(1-W \bar{W}_{1}\right)\left(1-W_{1} \bar{W}_{1}\right)^{-1} z_{1}+\left(W-W_{1}\right)\left(1-\bar{W}_{1} W_{1}\right)^{-1} \bar{z}_{1}\right], \\
W^{\prime}= & U\left(1-W_{1} \bar{W}_{1}\right)^{-1 / 2}\left(W-W_{1}\right)\left(1-\bar{W}_{1} W\right)^{-1}\left(1-\bar{W}_{1} W_{1}\right)^{1 / 2} U^{t},
\end{aligned}
$$

where $U$ is an unitary matrix. We find that

$$
\begin{aligned}
& \frac{\partial z^{\prime}}{\partial z}=U\left(1-W_{1} \bar{W}_{1}\right)^{1 / 2}\left(1-W \bar{W}_{1}\right)^{-1}, \\
& \mathrm{~d} W^{\prime}=A \mathrm{~d} W A^{t}, \quad A=U\left(1-W_{1} \bar{W}_{1}\right)^{1 / 2}\left(1-W \bar{W}_{1}\right)^{-1} .
\end{aligned}
$$

In order to calculate the Jacobian of the transformation (A.3), we use the following property extracted from Berezin's paper [20, p. 398]:

Let $A$ be a matrix and $L_{A}$ the transformation of a matrix of the same order $n, L_{A} \xi=A \xi A^{t}$. If the matrices $A$ and $\xi$ are symmetric, then $\operatorname{det} L_{A}=(\operatorname{det} A)^{n+1}$. We find as in [7], in accord with formula before Theorem 4.3.2 in [38]

$$
Q_{\mathcal{D}_{n}}=\operatorname{det}\left(\mathbb{1}_{n}-W \bar{W}\right)^{-(n+1)},
$$

while $Q_{\mathcal{D}_{n}^{J}}(z, W)$ has the expression (3.11).

\section{B Appendix}

\section{Laplace-Beltrami operator on the Siegel upper half-plan and Siegel ball}

The Laplace-Beltrami operator on $X_{n}$ has the expression (see [57, equation (19)])

$$
\Delta_{X_{n}}=-\operatorname{Tr}(Z-W)\left((Z-W) D_{w}\right)^{t} D_{z}, \quad Z=X+\mathrm{i} Y \in X_{n}, \quad W=\bar{Z},
$$

where we use the notation (3.39). Formula (43) in [57] reads

$$
D_{w}(Z-W)=-\frac{n+1}{2} \mathbb{1}_{n}+\left((Z-W) D_{w}\right)^{t},
$$

where

$$
\begin{aligned}
D_{w}(Z-W) & =\sum_{\rho=1}^{n} \mathrm{e}_{\mu \rho} \frac{\partial}{\partial w_{\mu \rho}}\left(z_{\rho \nu}-w_{\rho \nu}\right) \\
& =\left(\sum_{\rho=1}^{n}\left(z_{\mu \rho}-w_{\mu \rho}\right) e_{\rho \nu} \frac{\partial}{\partial w_{\rho \nu}}\right)^{t}-\left(\delta_{\mu \nu} \sum_{\rho=1}^{n} e_{\mu \rho}\right) .
\end{aligned}
$$


With formula (2.26), introducing in (B.3), it is obtained

$$
\left((Z-W) D_{w}\right)_{\mu \nu}^{t}(f)=\left[\frac{\partial f}{\partial W}(Z-W)\right]_{\mu \nu}=\left(z_{\rho \nu}-w_{\rho \nu}\right) e_{\mu \rho} \frac{\partial f}{\partial w_{\mu \rho}} .
$$

Equation (54) of the Cayley transform [57] in the notation (B.2) reads

$$
\tilde{Z}=\left(Z-\mathrm{i} \mathbb{1}_{n}\right)\left(Z+\mathrm{i} \mathbb{1}_{n}\right)^{-1} .
$$

Equation (55) in [57] asserts that

$$
D_{z}=-\frac{\mathrm{i}}{2}\left(\tilde{Z}-\mathbb{1}_{n}\right)\left(\left(\tilde{Z}-\mathbb{1}_{n}\right) D_{\tilde{z}}\right)^{t},
$$

i.e., if now $W$ describes a point (1.2) in $\mathcal{D}_{n}$, we have the formula

\section{Lemma B.1.}

$$
\begin{aligned}
\left(D_{z}\right)_{\alpha \beta} & :=e_{\alpha \beta} \frac{\partial}{\partial z_{\alpha \beta}}=-\frac{\mathrm{i}}{2}\left[\left(\mathbb{1}_{n}-W\right)\left(\left(\mathbb{1}_{n}-W\right) D_{w}\right)^{t}\right]_{\alpha \beta} \\
& =-\frac{\mathrm{i}}{2}\left(\mathbb{1}_{n}-W\right)_{\alpha \gamma}\left(\mathbb{1}_{n}-W\right)_{\beta \lambda} e_{\lambda \gamma} \frac{\partial}{\partial w_{\lambda \gamma}} .
\end{aligned}
$$

Proof. We write (2.21) as

$$
2 \mathrm{id} W=A \mathrm{~d} Z A, \quad A=\mathbb{1}_{n}-W, \quad W=W^{t}, \quad Z=Z^{t},
$$

i.e.,

$$
2 \mathrm{id} w_{p q}=A_{p m} \mathrm{~d} z_{m n} A_{n q} .
$$

With formula (2.26), we get

$$
\begin{aligned}
2 \mathrm{i} \frac{\partial w_{p q}}{\partial z_{\alpha \beta}} & =A_{p m} A_{n q} \frac{\partial z_{m n}}{\partial z_{\alpha \beta}}=A_{p m} A_{n q}\left(\delta_{m \alpha} \delta_{n \beta}+\delta_{m \beta} \delta_{n \alpha}-\delta_{m n} \delta_{\alpha \beta} \delta_{m \alpha}\right) \\
& =A_{p \alpha} A_{\beta q}+A_{p \beta} A_{\alpha q}-A_{p \alpha} A_{\beta q} \delta_{\alpha \beta} .
\end{aligned}
$$

We have the formula

$$
\frac{\partial f}{\partial z_{\alpha \beta}}=\sum_{p \leq q} \frac{\partial f}{\partial w_{p q}} \frac{\partial w_{p q}}{\partial z_{\alpha \beta}} .
$$

For $\alpha \neq \beta$, we get from (B.5):

$$
\begin{aligned}
2 \mathrm{i} \frac{\partial f}{\partial z_{\alpha \beta}} & =\sum_{p<q}\left(A_{p \alpha} A_{\beta q}+A_{p \beta} A_{\alpha q}\right) \frac{\partial f}{\partial w_{p q}}+\sum_{p=q}\left(A_{p \alpha} A_{\beta p}+A_{p \beta} A_{\alpha p}\right) \frac{\partial f}{\partial w_{p q}} \\
& =\sum_{p<q} A_{p \alpha} A_{\beta q} \frac{\partial f}{\partial w_{p q}}+\sum_{q<p} A_{q \beta} A_{\alpha p} \frac{\partial f}{\partial w_{p q}}+2 \sum_{p=q} A_{p \alpha} A_{\beta q} \frac{\partial f}{\partial w_{p q}} \delta_{p q} \\
& =\sum_{p q} A_{p \alpha} A_{\beta q}\left(1+\delta_{p q}\right) \frac{\partial f}{\partial w_{p q}} .
\end{aligned}
$$

Similarly, for $\alpha=\beta$, we have

$$
2 \mathrm{i} \frac{\partial f}{\partial z_{\alpha \beta}}=\sum_{p \leq q} A_{p \alpha} A_{\beta q} \frac{\partial f}{\partial w_{p q}}
$$




$$
\begin{aligned}
& =\frac{1}{2} \sum_{p<q} A_{p \alpha} A_{\beta q} \frac{\partial f}{\partial w_{p q}}+\frac{1}{2} \sum_{q<p} A_{q \alpha} A_{\beta p} \frac{\partial f}{\partial w_{q p}} \frac{1}{2}+\sum_{p=q} A_{p \alpha} A_{\beta q} \frac{\partial f}{\partial w_{p q}} \\
& =\frac{1}{2} \sum_{p \neq q} A_{p \alpha} A_{\beta q} \frac{\partial f}{\partial w_{p q}}+\sum_{p=q} A_{p \alpha} A_{\beta q} \frac{\partial f}{\partial w_{p q}}=\sum_{p q} A_{p \alpha} A_{\beta q} \frac{1+\delta_{p q}}{2} \frac{\partial f}{\partial w_{p q}} .
\end{aligned}
$$

We put together (B.6) and (B.7) as in (B.4).

With Lemma B.1, it is obtained the Laplace-Beltrami operator on the Siegel ball (see [57, equation (56)])

Proposition B.2. The Laplace-Beltrami operator on the Siegel ball $\mathcal{D}_{n}$ has the expression

$$
\begin{aligned}
& \Delta_{\mathcal{D}_{n}}(W)=\operatorname{Tr}\left[N\left(N D_{\bar{W}}\right)^{t} D_{W}\right]=\sum_{p, q, m, n} \mathbb{K}_{p q \bar{m} \bar{n}} \frac{\partial^{2}}{\partial \bar{w}_{m n} \partial w_{p q}}, \quad N:=\mathbb{1}_{n}-W \bar{W}, \\
& \mathbb{K}_{p q \bar{m} \bar{n}}=e_{m n} e_{p q} K_{p q \bar{m} \bar{n}}, \quad K_{p q \bar{m} \bar{n}}=N_{q n} \bar{N}_{m p},
\end{aligned}
$$

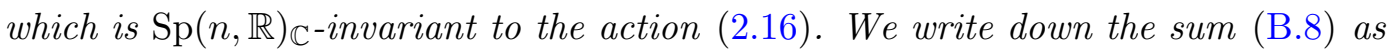

$$
\Delta_{\mathcal{D}_{n}}(W)=\sum_{m \leq n ; p \leq q} k_{m n \bar{p} \bar{q}}(W) \frac{\partial^{2}}{\partial \bar{w}_{m n} \partial w_{p q}},
$$

where, with the expression (B.9), we have

$$
\begin{aligned}
& k_{p q \bar{m} \bar{n}}=\frac{1}{4}\left(K_{p q \bar{m} \bar{n}}+K_{q p \bar{m} \bar{n}}+K_{p q \bar{n} \bar{m}}+K_{q p \bar{n} \bar{m}}\right), \quad m<n, \quad p<q, \\
& k_{p q \bar{m} \bar{m}}=\frac{1}{2}\left(K_{p q \bar{m} \bar{m}}+K_{q p \bar{m} \bar{m}}\right), \quad p<q, \quad n=m, \\
& k_{p p \bar{m} \bar{n}}=\frac{1}{2}\left(K_{p p \bar{m} \bar{n}}+K_{p p \bar{n} \bar{m}}\right), \quad p=q, \quad m<n, \\
& k_{p p \bar{m} \bar{m}}=K_{p p \bar{m} \bar{m}} .
\end{aligned}
$$

(B.10) can be written down as

$$
\begin{aligned}
k_{m n \bar{p} \bar{q}} & =e_{m n}\left(1-\delta_{p q}\right) N_{p m} \bar{N}_{n q}+e_{p q}\left(1-\delta_{m n}\right) N_{q m} \bar{N}_{n p}+\delta_{p q} \delta_{m n} N_{p m} \bar{N}_{n q} \\
& =\frac{1}{2}\left(N_{q n} \bar{N}_{m p}+N_{q m} \bar{N}_{n p}\right) .
\end{aligned}
$$

Proof. With equation (B.4) introduced in (B.1), it is obtained (B.8).

In order to directly (i.e., without Remark A.1) prove the invariance of $\Delta_{\mathcal{D}_{n}}$ to the group action $g \times W=W_{1}, g \in \operatorname{Sp}(n, \mathbb{R})_{C}, W, W_{1} \in \mathcal{D}_{n}$, we use the formulas (3.28), (3.29). We write $(3.28)$ as

$$
\mathrm{d} w_{p q}=A_{p m}\left(\mathrm{~d} w_{1}\right)_{m n} A_{q n}, \quad \text { where } \quad A=W q^{*}+p^{*} .
$$

With formula (2.26), we have successively

$$
\begin{aligned}
\frac{\partial w_{p q}}{\partial\left(w_{1}\right)_{\alpha \beta}} & =A_{p m} \frac{\partial\left(w_{1}\right)_{m n}}{\partial\left(w_{1}\right)_{\alpha \beta}} A_{n q}=A_{p m} A_{n q} \Delta_{\alpha \beta}^{m n} \\
& =A_{p \alpha} A_{q \beta}+A_{p \beta} A_{q \alpha}-\delta_{\alpha \beta} A_{p \alpha} A_{q \beta} .
\end{aligned}
$$

With (B.12), we calculate $\frac{\partial f}{\partial\left(w_{1}\right)_{\alpha \beta}}$. 
a) Firstly, we consider the case $\alpha \neq \beta$. We have

$$
\begin{aligned}
\frac{\partial f}{\partial\left(w_{1}\right)_{\alpha \beta}} & =\left(\sum_{p<q} A_{p \alpha} A_{q \beta}+\sum_{q<p} A_{q \alpha} A_{p \beta}+2 \sum_{p=q} A_{p \alpha} A_{q \beta}\right) \frac{\partial f}{\partial w_{p q}} \\
& =2 \sum A_{p \alpha} A_{q \beta} e_{p q} \frac{\partial f}{\partial w_{p q}} .
\end{aligned}
$$

b) Now we consider the case $\alpha=\beta$. We have

$$
\frac{\partial f}{\partial\left(w_{1}\right)_{\alpha \beta}}=2 f_{\alpha \beta} \sum_{p, q} A_{p \alpha} A_{q \beta} e_{p q} .
$$

(B.13) and (B.14) are written together as

$$
D_{W_{1}}=A^{t} D_{W} A \text {. }
$$

Also we write (3.29) as

$$
M_{1}=\bar{A}^{t} M A \text {. }
$$

With (B.15) and (B.16) introduced in formula (B.8), we prove

$$
\Delta_{\mathcal{D}_{n}}\left(W_{1}\right)=\Delta_{\mathcal{D}_{n}}(W), \quad \text { where } \quad W_{1}=g \times W .
$$

Remark B.3. In the notation of [39]

$$
Z=\left(\begin{array}{cccc}
\sqrt{2} z_{11} & z_{12} & \ldots & z_{1 n} \\
z_{12} & \sqrt{2} z_{22} & \ldots & z_{2 n} \\
\cdots & \cdots & \cdots & \cdots \\
z_{1 n} & z_{2 n} & \cdots & \sqrt{2} z_{n n}
\end{array}\right), \quad \partial_{Z}=\left(\begin{array}{cccc}
\sqrt{2} \frac{\partial}{\partial z_{11}} & \frac{\partial}{\partial z_{12}} & \cdots & \frac{\partial}{\partial z_{1 n}} \\
\frac{\partial}{\partial z_{12}} & \sqrt{2} \frac{\partial}{\partial z_{22}} & \cdots & \frac{\partial}{\partial z_{2 n}} \\
\cdots & \cdots & \cdots & \cdots \\
\frac{\partial}{\partial z_{1 n}} & \frac{\partial}{\partial z_{2 n}} & \cdots & \sqrt{2} \frac{\partial}{\partial z_{n n}}
\end{array}\right)
$$

the Laplace-Beltrami operator reads (see [39, equation (2.6.4)])

$$
\Delta_{\mathcal{D}_{n}}(W)=\operatorname{Tr}\left(\left(\mathbb{1}_{n}-\bar{W} W\right) \partial_{W}\left(\mathbb{1}_{n}-W \bar{W}\right) \bar{\partial}_{W}\right),
$$

i.e.,

$$
\begin{aligned}
\Delta_{\mathcal{D}_{n}}(W)= & \sum_{\alpha \beta \lambda \mu=1}^{n}\left(\delta_{\lambda \mu}-\sum_{\sigma=1}^{n} P_{\lambda \sigma} P_{\mu \sigma} w_{\lambda \sigma} \bar{w}_{\mu \sigma}\right) \\
& \times\left(\delta_{\alpha \beta}-\sum_{\gamma=1}^{n} P_{\alpha \gamma} P_{\beta \gamma} w_{\alpha \gamma} \bar{w}_{\beta \gamma}\right) P_{\lambda \alpha} P_{\mu \beta} \frac{\partial^{2}}{\partial w_{\lambda \alpha} \partial \bar{w}_{\mu \beta}},
\end{aligned}
$$

where

$$
P_{\alpha \beta}=1+(\sqrt{2}-1) \delta_{\alpha \beta} .
$$

" $P$ " is related with the symbol " $e "$ in (3.39) by the relation

$$
P_{\alpha \beta}=\sqrt{2}(\sqrt{2}-1)\left(1+\sqrt{2} e_{\alpha \beta}\right) .
$$

\section{Acknowledgements}

I am grateful to Daniel Beltita for clarifying some aspects of contractibility related with this paper. This research was conducted in the framework of the ANCS project program PN 1642 01 01/2016 and UEFISCDI-Romania program PN-II-PCE-55/05.10.2011. 


\section{References}

[1] Arezzo C., Loi A., Moment maps, scalar curvature and quantization of Kähler manifolds, Comm. Math. Phys. 246 (2004), 543-559.

[2] Berceanu S., Coherent states and geodesics: cut locus and conjugate locus, J. Geom. Phys. 21 (1997), 149-168, dg-ga/9502007.

[3] Berceanu S., On the geometry of complex Grassmann manifold, its noncompact dual and coherent states, Bull. Belg. Math. Soc. Simon Stevin 4 (1997), 205-243, dg-ga/9509002.

[4] Berceanu S., Realization of coherent state Lie algebras by differential operators, in Advances in Operator Algebras and Mathematical Physics, Theta Ser. Adv. Math., Vol. 5, Editors F. Boca, O. Bratteli, R. Longo, H. Siedentop, Theta, Bucharest, 2005, 1-24, math.DG/0504053.

[5] Berceanu S., A holomorphic representation of the Jacobi algebra, Rev. Math. Phys. 18 (2006), 163-199, Errata, Rev. Math. Phys. 24 (2012), 1292001, 2 pages, math.DG/0408219.

[6] Berceanu S., A holomorphic representation of the semidirect sum of symplectic and Heisenberg Lie algebras, J. Geom. Symmetry Phys. 5 (2006), 5-13.

[7] Berceanu S., A holomorphic representation of the multidimensional Jacobi algebra, in Perspectives in Operator Algebras and Mathematical Physics, Theta Ser. Adv. Math., Vol. 8, Editors F.-P. Boca, R. Purice, S. Stratila, Theta, Bucharest, 2008, 1-25, math.DG/0604381.

[8] Berceanu S., The Jacobi group and the squeezed states - some comments, AIP Conf. Proc. 1191 (2009), 21-29, arXiv:0910.5563.

[9] Berceanu S., A convenient coordinatization of Siegel-Jacobi domains, Rev. Math. Phys. 24 (2012), 1250024, 38 pages, arXiv:1204.5610.

[10] Berceanu S., Coherent states and geometry on the Siegel-Jacobi disk, Int. J. Geom. Methods Mod. Phys. 11 (2014), 1450035, 25 pages, arXiv:1307.4219.

[11] Berceanu S., Quantum mechanics and geometry on the Siegel-Jacobi disk, in Geometric Methods in Physics, Editors P. Kielanowski, P. Bieliavsky, A. Odesski, A. Odzijewicz, M. Schlichenmaier, T. Voronov, Trends in Mathematics, Birkhäuser, Basel, 2014, 89-98.

[12] Berceanu S., Bergman representative coordinates on the Siegel-Jacobi disk, Romanian J. Phys. 60 (2015), 867-896, arXiv:1409.0368.

[13] Berceanu S., Gheorghe A., Differential operators on orbits of coherent states, Romanian J. Phys. 48 (2003), 545-556, math.DG/0211054.

[14] Berceanu S., Gheorghe A., Applications of the Jacobi group to quantum mechanics, Romanian J. Phys. 53 (2008), 1013-1021, arXiv:0812.0448.

[15] Berceanu S., Gheorghe A., On the geometry of Siegel-Jacobi domains, Int. J. Geom. Methods Mod. Phys. 8 (2011), 1783-1798, arXiv:1011.3317.

[16] Berceanu S., Schlichenmaier M., Coherent state embeddings, polar divisors and Cauchy formulas, J. Geom. Phys. 34 (2000), 336-358, math.DG/9903105.

[17] Berezin F.A., Quantization in complex bounded domains, Dokl. Akad. Nauk SSSR 211 (1973), $1263-1266$.

[18] Berezin F.A., Quantization, Izv. Akad. Nauk SSSR Ser. Mat. 38 (1974), 1116-1175.

[19] Berezin F.A., General concept of quantization, Comm. Math. Phys. 40 (1975), 153-174.

[20] Berezin F.A., Quantization in complex symmetric spaces, Izv. Akad. Nauk SSSR Ser. Mat. 39 (1975), 363-402.

[21] Berndt R., Sur l'arithmétique du corps des fonctions elliptiques de niveau $N$, in Seminar on Number Theory, Paris 1982-83 (Paris, 1982/1983), Progr. Math., Vol. 51, Birkhäuser Boston, Boston, MA, 1984, 21-32.

[22] Berndt R., Böcherer S., Jacobi forms and discrete series representations of the Jacobi group, Math. Z. 204 (1990), 13-44.

[23] Berndt R., Schmidt R., Elements of the representation theory of the Jacobi group, Progr. Math., Vol. 163, Birkhäuser Verlag, Basel, 1998.

[24] Borel A., Kählerian coset spaces of semisimple Lie groups, Proc. Nat. Acad. Sci. USA 40 (1954), 1147-1151.

[25] Cahen B., Global parametrization of scalar holomorphic coadjoint orbits of a quasi-Hermitian Lie group, Acta Univ. Palack. Olomuc. Fac. Rerum Natur. Math. 52 (2013), 35-48.

[26] Cahen B., Stratonovich-Weyl correspondence for the Jacobi group, Commun. Math. 22 (2014), 31-48. 
[27] Cahen M., Gutt S., Rawnsley J., Quantization of Kähler manifolds. II, Trans. Amer. Math. Soc. 337 (1993), 73-98.

[28] Calabi E., Isometric imbedding of complex manifolds, Ann. of Math. 58 (1953), 1-23.

[29] Chern S.S., Complex manifolds without potential theory, 2nd ed., Universitext, Springer-Verlag, New York Heidelberg, 1979.

[30] Chiribella G., Adesso G., Quantum benchmarks for pure single-mode Gaussian states, Phys. Rev. Lett. 112 (2014), 010501, 6 pages, arXiv:1308.2146.

[31] Donaldson S.K., Scalar curvature and projective embeddings. I, J. Differential Geom. 59 (2001), 479-522.

[32] Dorfmeister J., Nakajima K., The fundamental conjecture for homogeneous Kähler manifolds, Acta Math. 161 (1988), 23-70.

[33] Eichler M., Zagier D., The theory of Jacobi forms, Progr. Math., Vol. 55, Birkhäuser Boston, Inc., Boston, MA, 1985.

[34] Engliš M., Berezin quantization and reproducing kernels on complex domains, Trans. Amer. Math. Soc. 348 (1996), 411-479.

[35] Gay-Balmaz F., Tronci C., Vlasov moment flows and geodesics on the Jacobi group, J. Math. Phys. 53 (2012), 123502, 36 pages, arXiv:1105.1734.

[36] Helgason S., Differential geometry, Lie groups, and symmetric spaces, Pure and Applied Mathematics, Vol. 80, Academic Press, Inc., New York - London, 1978.

[37] Hua L.-K., On the theory of automorphic functions of a matrix level. I. Geometrical basis, Amer. J. Math. 66 (1944), 470-488.

[38] Hua L.-K., Harmonic analysis of functions of several complex variables in the classical domains, Amer. Math. Soc., Providence, R.I., 1963.

[39] Hua L.-K., Look K.H., Theory of harmonic functions in classical domains, Scientia Sinica 8 (1959), 743-806.

[40] Hunziker M., Sepanski M.R., Stanke R.J., Global Lie symmetries of a system of Schrödinger equations and the oscillator representation, Miskolc Math. Notes 14 (2013), 647-657.

[41] Jost J., Riemannian geometry and geometric analysis, 5th ed., Universitext, Springer-Verlag, Berlin, 2008.

[42] Kähler E., Raum-Zeit-Individuum, Rend. Accad. Naz. Sci. XL Mem. Mat. (5) 16 (1992), $115-177$.

[43] Kähler E., Mathematische Werke/Mathematical works, Walter de Gruyter \& Co., Berlin, 2003.

[44] Kobayashi S., Geometry of bounded domains, Trans. Amer. Math. Soc. 92 (1959), 267-290.

[45] Kobayashi S., Nomizu K., Foundations of differential geometry. Vol. I, Interscience Publishers, New York London, 1963.

[46] Kobayashi S., Nomizu K., Foundations of differential geometry. Vol. II, Interscience Publishers, New York London - Sydney, 1969.

[47] Kostant B., Quantization and unitary representations. I. Prequantization, in Lectures in Modern Analysis and Applications, III, Lecture Notes in Math., Vol. 170, Springer, Berlin, 1970, 87-208.

[48] Kramer P., Saraceno M., Semicoherent states and the group ISp(2, R), Phys. A 114 (1982), 448-453.

[49] Lee M.H., Theta functions on Hermitian symmetric domains and Fock representations, J. Aust. Math. Soc. 74 (2003), 201-234.

[50] Lisiecki W., Coherent state representations. A survey, Rep. Math. Phys. 35 (1995), 327-358.

[51] Loi A., Regular quantizations of Kähler manifolds and constant scalar curvature metrics, J. Geom. Phys. 53 (2005), 354-364.

[52] Loi A., Mossa R., Berezin quantization of homogeneous bounded domains, Geom. Dedicata 161 (2012), 119-128, arXiv:1106.2510.

[53] Loi A., Mossa R., Some remarks on homogeneous Kähler manifolds, Geom. Dedicata 179 (2015), 377-383, arXiv:1502.00011.

[54] Lu Q.-K., On Kähler manifolds with constant curvature, Acta. Math. Sin. 66 (1966), 269-281.

[55] Lu Q.-K., Holomorphic invariant forms of a bounded domain, Sci. China Ser. A 51 (2008), 1945-1964.

[56] Lütkepohl H., Handbook of matrices, John Wiley \& Sons, Ltd., Chichester, 1996.

[57] Maass H., Die Differentialgleichungen in der Theorie der Siegelschen Modulfunktionen, Math. Ann. 126 (1953), 44-68. 
[58] Mandel L., Wolf E., Optical coherence and quantum optics, Cambridge University Press, Cambridge, 1995.

[59] Marmo G., Michor P.W., Neretin Yu.A., The Lagrangian Radon transform and the Weil representation, J. Fourier Anal. Appl. 20 (2014), 321-361, arXiv:1212.4610.

[60] Molitor M., Gaussian distributions, Jacobi group, and Siegel-Jacobi space, J. Math. Phys. 55 (2014), 122102, 40 pages, arXiv:1409.7917.

[61] Moroianu A., Lectures on Kähler geometry, London Mathematical Society Student Texts, Vol. 69, Cambridge University Press, Cambridge, 2007.

[62] Neeb K.-H., Holomorphy and convexity in Lie theory, de Gruyter Expositions in Mathematics, Vol. 28, Walter de Gruyter \& Co., Berlin, 2000.

[63] Nomizu K., Invariant affine connections on homogeneous spaces, Amer. J. Math. 76 (1954), 33-65.

[64] Perelomov A., Generalized coherent states and their applications, Texts and Monographs in Physics, Springer-Verlag, Berlin, 1986.

[65] Quesne C., Vector coherent state theory of the semidirect sum Lie algebras wsp(2N, R), J. Phys. A: Math. Gen. 23 (1990), 847-862.

[66] Rawnsley J., Cahen M., Gutt S., Quantization of Kähler manifolds. I. Geometric interpretation of Berezin's quantization, J. Geom. Phys. 7 (1990), 45-62.

[67] Rawnsley J.H., Coherent states and Kähler manifolds, Quart. J. Math. Oxford Ser. (2) 28 (1977), $403-415$.

[68] Satake I., Algebraic structures of symmetric domains, Kanô Memorial Lectures, Vol. 4, Iwanami Shoten, Tokyo, Princeton University Press, Princeton, N.J., 1980.

[69] Sepanski M.R., Stanke R.J., Global Lie symmetries of the heat and Schrödinger equation, J. Lie Theory 20 (2010), 543-580.

[70] Shuman K.L., Complete signal processing bases and the Jacobi group, J. Math. Anal. Appl. 278 (2003), 203-213.

[71] Siegel C.L., Symplectic geometry, Amer. J. Math. 65 (1943), 1-86.

[72] Takase K., A note on automorphic forms, J. Reine Angew. Math. 409 (1990), 138-171.

[73] Takase K., On unitary representations of Jacobi groups, J. Reine Angew. Math. 430 (1992), 139-149.

[74] Takase K., On Siegel modular forms of half-integral weights and Jacobi forms, Trans. Amer. Math. Soc. 351 (1999), 735-780.

[75] Vinberg E.B., Gindikin S.G., Kähler manifolds admitting a transitive solvable group of automorphisms, Math. USSR Sb. 3 (1967), 333-351.

[76] Yang J.-H., The method of orbits for real Lie groups, Kyungpook Math. J. 42 (2002), 199-272, math.RT/0602056.

[77] Yang J.-H., Invariant metrics and Laplacians on Siegel-Jacobi space, J. Number Theory 127 (2007), 83-102, math.NT/0507215.

[78] Yang J.-H., A partial Cayley transform of Siegel-Jacobi disk, J. Korean Math. Soc. 45 (2008), 781-794, math.NT/0507216.

[79] Yang J.-H., Invariant metrics and Laplacians on Siegel-Jacobi disk, Chin. Ann. Math. Ser. B 31 (2010), 85-100, math.NT/0507217.

[80] Yang J.-H., Yong Y.-H., Huh S.-N., Shin J.-H., Min G.-H., Sectional survatures of the Siegel-Jacobi space, Bull. Korean Math. Soc. 50 (2013), 787-799. 